\title{
Auf dem Weg zur digitalen Nahversorgung? Determinanten des Einkaufsverhaltens im Multi- und Cross-Channel-Kontext am Fallbeispiel des Lebensmitteleinzelhandels
}

\author{
Thomas Wieland (1) \\ Eingegangen: 4. September 2020 - Angenommen: 28. Januar 2021 - Online veröffentlicht: 26. Februar 2021
}

\section{Zusammenfassung}

Die Multi- bzw. Cross-Channel-Einbindung des deutschen Lebensmitteleinzelhandels zeigt sich an der Etablierung onlinegestützter Lieferdienste und dem Angebot des Click-andcollect-Services durch bestehende Verbrauchermärkte. Vor diesem Hintergrund stellt sich die Frage, inwiefern der reine Lebensmittelonlinehandel bzw. Cross-Channeling im stationären Handel eine Ergänzung bisheriger Vertriebswege in der Nahversorgung darstellen kann. Dieser Beitrag beschreibt die Untersuchung der wichtigsten Determinanten des räumlichen Einkaufsverhaltens im Lebensmitteleinzelhandel. Insbesondere wurde geprüft, ob Lebensmittelmärkte von der Clickand-collect-Option profitieren können und welche Faktoren die Einkaufsstättenwahl bedingen, wenn sowohl stationäre als auch Online-Anbieter zur Verfügung stehen. Als empirischer Zugang zur Beantwortung dieser Forschungsfragen fungiert die durch ein mikroökonometrisches Modell der Einkaufsstättenwahl (Hurdle-Modell) vorgenommene Analyse repräsentativer Konsumentenbefragungen in zwei deutschen Regionen (Südniedersachsen, Region Mittlerer Oberrhein). Es zeigt sich, dass der Lebensmittelonlinehandel nur eine sehr geringe Relevanz hat und das Click-and-collect-Angebot keinen Einfluss auf die Kundenanziehungskraft von Verbrauchermärkten hat. Die Wahrscheinlichkeit des LebensmittelOnlinekaufs wird vorrangig durch psychographische Merkma-

Dr. Thomas Wieland, Institut für Geographie und Geoökologie, Karlsruher Institut für Technologie, Kaiserstraße 12, 76131 Karlsruhe thomas.wieland@kit.edu

c 2021 Wieland; licensee oekom verlag. This Open Access article is published under the Creative Commons Attribution-ShareAlike 4.0 International Licence. le der Konsumenten erklärt. Im direkten Vergleich erweisen sich raumbezogene Transaktionskosten (Fahrtzeit, Versandkosten) als wichtige Determinanten der Einkaufsstätten- bzw. Kanalwahl.

Schlüsselwörter: Lebensmitteleinzelhandel ·

Nahversorgung · Onlinehandel · Click and collect · Modell der Einkaufsstättenwahl

Digital local supply on the rise? Determinants of multi- and cross-channel shopping behaviour in grocery retailing

\section{Abstract}

Multi- and cross-channel integration of German grocery retailing appears in terms of online-based delivery services and click-and-collect offers by established supermarkets. However, these trends raise the question whether digital services may complement established distribution channels, especially with respect to local supply. This paper describes the investigation of the main determinants of spatial shopping behaviour in the food retail sector. In particular, it was examined whether grocery stores can benefit from the clickand-collect option and which factors determine the choice of shopping location if both stationary and online providers are available.

Individual store choices and the related expenditures at grocery retailers are investigated using a micro-econometric store choice model (hurdle model) based on a representative customer survey in two German regions (South Lower Saxony, Region Middle Upper Rhine). Pure grocery online retailing is found to have little relevance, whilst supermarkets gain little profit from offering the click-and-collect option. The likeli- 
hood of buying groceries online can be explained by psychographic characteristics of the consumers. In a situation where consumers may choose between offline and online retailers, channel-specific transaction costs (driving time, shipping charges) can be identified as important determinants of store choice.

Keywords: Grocery retailing - Local supply · Online retailing $\cdot$ Click and collect $\cdot$ Store choice model

\section{Einleitung}

Der Lebensmitteleinzelhandel hatte im Jahr 2017 mit einem Umsatz von 187 Mrd. Euro den größten Anteil (36,4\%) am deutschen Einzelhandel (HDE/IFH 2018: 34). Er ist zudem ein integraler Bestandteil der Nahversorgung, deren Aufrechterhaltung bzw. Ausbau eine normative Zielvorstellung in der räumlichen Planung ist (Küpper/Scheibe 2015: 46). Gerade deswegen sind Neuansiedlungen oder Erweiterungen von bestehenden Lebensmittelmärkten immer wieder Gegenstand von Verträglichkeitsprüfungen, deren Ergebnis die Entscheidungen im Rahmen der kommunalen Bauleitplanung bzw. Raumordnung beeinflussen (Anders 2018: 298-300; Müller-Hagedorn 2020: 106-116).

Abgesehen von der dynamischen Betriebsformen- und Standortentwicklung schreitet auch die Multi- und CrossChannel-Einbindung im Lebensmitteleinzelhandel voran. Unter Multi-Channeling wird der parallele, jedoch voneinander unabhängige Einsatz von mehreren, den Kunden zur Verfügung stehenden Kauf- und Informationskanälen durch ein Einzelhandelsunternehmen verstanden. Demgegenüber umfasst Cross-Channeling die Verbindung mehrerer Vertriebskanäle, sodass Kunden im Rahmen desselben Kaufprozesses mehrere dieser Kanäle nutzen können (Rittinger 2013: 15-22). Im deutschen Lebensmitteleinzelhandel zeigen sich drei Entwicklungen:

- Der weltgrößte Non-Food-Onlinehändler Amazon hat mittlerweile Lebensmittel ins Liefersortiment aufgenommen.

- Filialisten bzw. Kooperativen des stationären Lebensmitteleinzelhandels haben separate Lieferdienste eröffnet, die jedoch nicht flächendeckend verfügbar sind (Rewe).

- Es zeigt sich eine zunehmende Cross-Channel-Integration durch die Einführung der Click-and-collect-Option bei einzelnen selbstständigen, aber in Kooperativen organisierten Märkten (vor allem Edeka, Rewe).

Der Lebensmittel-Onlinehandel hat im Jahr 2019 einen Marktanteil am gesamten Lebensmitteleinzelhandel von 1,4\% erreicht; die Tendenz ist steigend (HDE/IFH 2020:
8). Ob der Lebensmittel-Onlinehandel eine ernstzunehmende Bedrohung für den stationären Lebensmitteleinzelhandel darstellt und als Folge eine weitere Ausdünnung des Verkaufsstellennetzes droht, erscheint vor diesem Hintergrund fraglich. Es verwundert aber nicht, wenn in Deutschland bereits die Möglichkeiten und Grenzen des Lebensmittel-Onlinehandels als ergänzende oder alternative Nahversorgung diskutiert werden (z. B. Dannenberg/Franz/Lepper 2016; Mensing/Neiberger 2018; Dannenberg/Dederichs 2019; Kokorsch/Küpper 2019). Eine andere Frage ist die Relevanz der Cross-Channel-Einbindung bestehender Lebensmittelmärkte. Kokorsch und Küpper (2019: 23) argumentieren etwa, dass der stationäre Lebensmitteleinzelhandel von der Einführung der Click-and-collect-Option profitieren könnte, was einer Umsatzverschiebung zugunsten des reinen Lebensmittel-Onlinehandels Vorschub leisten und bestehende Nahversorgungsstrukturen stärken könnte.

Dieser Beitrag geht im Rahmen einer empirischen Untersuchung folgenden Fragestellungen nach:

- Welche sind die wichtigsten Determinanten des räumlichen Einkaufsverhaltens im Lebensmitteleinzelhandel, das heißt, welche angebots- und/oder nachfrageseitigen Attribute erklären die Einkaufsstättenwahl? Hierbei sollen traditionelle Erklärungsansätze zur Erreichbarkeit, zum Sortiment und zu Agglomerationseffekten berücksichtigt und um bisher nicht behandelte Aspekte erweitert werden, die sich auf die Multi- bzw. Cross-Channel-Integration des Lebensmitteleinzelhandels beziehen:

- Können Lebensmittelmärkte von der Click-and-collectOption im Hinblick auf ihre Kundenzuflüsse profitieren?

- Welche Faktoren bedingen die Einkaufsstättenwahl im Lebensmitteleinzelhandel, wenn sowohl stationäre als auch Online-Anbieter zur Verfügung stehen? Welche Rolle spielen insbesondere die Erreichbarkeit stationärer Lebensmittelmärkte und die Liefergebühr von Lebensmittel-Onlinehändlern sowie demographische und einstellungsbezogene Eigenschaften der Konsumentinnen und Konsumenten?

Als empirischer Zugang zur Beantwortung dieser Forschungsfragen fungiert die durch ein Modell der Einkaufsstättenwahl vorgenommene Analyse repräsentativer Befragungen in zwei deutschen Regionen. Die Studie soll neue empirische Erkenntnisse zum raumrelevanten Einkaufsverhalten im Lebensmitteleinzelhandel liefern, die sowohl für (Handels-)Unternehmen als auch für die Praxis der Raumordnung und Stadtplanung relevant sind. Letztgenannte Instanzen haben die Aufrechterhaltung funktionierender Nahversorgung zur Aufgabe, wobei sich die Frage stellt, inwieweit Multi- bzw. Cross-Channeling im Lebensmittel- 
einzelhandel die bestehende Standortlandschaft zukünftig verändert.

Da sich dieser Beitrag auf das Konsumentenverhalten beim Lebensmitteleinkauf fokussiert, wird zunächst der Stand der Forschung zum Einkaufsverhalten im stationären Lebensmitteleinzelhandel und zur Kanalwahl im MultiChannel-Kontext aufgearbeitet (Kapitel 2). Diese bisherigen Erkenntnisse bilden den Ausgangspunkt für den eigenen empirischen Ansatz, der im darauffolgenden Kapitel 3 vorgestellt wird. Im Ergebnisteil (Kapitel 4) erfolgt zunächst eine deskriptive Darstellung wichtiger Teilergebnisse und danach die Gegenüberstellung der Modellergebnisse zum räumlichen Einkaufsverhalten. Der Beitrag schließt mit einer Zusammenstellung der Schlussfolgerungen (Kapitel 5) $a b$.

\section{Stand der Forschung zu den Determinanten des (räumlichen) Einkaufsverhaltens}

\subsection{Räumliches Einkaufsverhalten im stationären Lebensmitteleinzelhandel}

Als wichtigste Wettbewerbsparameter im Lebensmitteleinzelhandel identifizieren Toporowski und Lademann (2014: 132) die Sortiments-, Preis- und Standortpolitik, weshalb sich die vorliegende Darstellung auf diese drei Erklärungsgrößen beschränkt. Der empirische Zugang zu den Determinanten des räumlichen Einkaufsverhaltens erfolgt in der Regel durch den Einsatz von quantitativen Modellen der Einkaufsstättenwahl, mit denen der Einfluss bestimmter Erklärungsgrößen auf das reale Einkaufsverhalten untersucht wird. Diesen Modellen liegen entweder empirische Marktgebiete von Standorten oder individuelle Kaufentscheidungen zugrunde, die mithilfe ökonometrischer Methoden (insbesondere Regressionsanalyse) den Erklärungsgrößen gegenübergestellt werden (Wieland 2015: 61-64).

Als theoretische und konzeptionelle Grundlage fungiert hier regelmäßig das von Huff (1962) entwickelte Marktgebietsmodell. Der Nutzen einer Einkaufsstätte für die Nachfrager wird darin durch zwei Variablen definiert: Einerseits wird ein überproportional negativer Einfluss der Wegezeit zwischen dem Kundenwohnort und dem Angebotsstandort auf den Kundenzufluss angenommen, was mit den Opportunitätskosten längerer Einkaufsfahrten begründet wird. Als zweite Determinante wird die Sortimentsbreite und -tiefe von Angebotsstandorten angenommen, die im Modell durch deren Verkaufsfläche operationalisiert wird und der ein degressiv-positiver Einfluss auf die Einkaufsstättenwahl zugeschrieben wird. Der positive Effekt wird damit begründet, dass die Einkaufsentscheidung unter unvollständiger Markt- übersicht getroffen wird und die potenziellen Kundinnen und Kunden nicht wissen, ob sämtliche gewünschten Produkte am jeweiligen Standort verfügbar sind; je größer allerdings das Sortiment, desto höher sei die Wahrscheinlichkeit eines ,erfolgreichen 'Einkaufs. Gleichzeitig steigen aber mit dem Angebot auch Such- und Entscheidungskosten der Nachfrager, weswegen von abnehmendem Grenznutzen ausgegangen wird, was den degressiv-positiven Effekt erklärt (Huff 1962: 16-19).

Tatsächlich wurde regelmäßig die Erreichbarkeit von Lebensmittelmärkten als zentrale oder gar wichtigste Erklärungsgröße der Einkaufsstättenwahl identifiziert: Die entweder als Entfernung oder als Fahrtzeit ausgedrückte Variable zeigt in modellgestützten Analysen stets einen (i.d.R. überproportional) negativen Einfluss auf die Kundenzuflüsse (Popkowski Leszczyc/Sinha/Sahgal 2004: 92-95; Lademann 2007: 155-156; Briesch/Chintagunta/Fox 2009: 183-187; Reutterer/Teller 2009: 704-706; Tihi/Oruc 2012: 44; Hillier/Smith/Whiteman et al. 2017: 6-8; Suárez-Vega/ Gutiérrez-Acuña/Rodríguez-Díaz 2015: 225-227; Wieland 2015: 173-177; Anders 2018: 303-304; Wieland 2018: 382-385). Die hohe Relevanz der Erreichbarkeit zeigt eine Marktgebietsanalyse von Wieland (2015: 175-176), in der die Hälfte der Varianz empirischer regionaler Marktanteile in einem Regressionsmodell (MCI-Modell) allein über die Pkw-Fahrtzeit erklärt wird und eine Standardisierung der Koeffizienten aufzeigt, dass diese das höchste Bedeutungsgewicht von allen untersuchten ErklärungsgröBen hat. Durch die Ermittlung von Elastizitäten zeigen Briesch, Chintagunta und Fox (2009: 186), dass beim Lebensmittelkauf die (negative) Konsumentenreaktion auf eine steigende Distanz zum Geschäft höher ist als bei den anderen untersuchten Wettbewerbsparametern. Ein ähnliches Ergebnis zeigt eine Modellanalyse zur individuellen Einkaufsstättenwahl von Wieland (2018: 384-385). In mehreren modellgestützten Studien wird nachgewiesen, dass der Erreichbarkeitseffekt zwischen Betriebsformen des Lebensmitteleinzelhandels (Briesch/Chintagunta/Fox 2009: 186; Anders 2018: 303-304), aber auch zwischen Kundentypen variiert (Popkowski Leszczyc/Sinha/Sahgal 2004: 92-95).

Gemäß der Annahme im Huff-Modell ist regelmäßig auch ein (degressiv-)positiver Effekt der Verkaufsflächengröße festgestellt worden (Lademann 2007: 155-156; Tihi/ Oruc 2012: 44; Suárez-Vega/Gutiérrez-Acuña/RodríguezDíaz 2015: 225-227; Wieland 2015: 173-177; Hillier/Smith/ Whiteman et al. 2017: 6-8; Wieland 2018: 382-385). Auch dieser Effekt zeigt sich als betriebsformenabhängig (Lademann 2007: 155-156; Wieland 2015: 180-182). Briesch, Chintagunta und Fox (2009: 186) verwenden in ihrem Modell der Einkaufsstättenwahl statt eines Proxys den tatsächlichen Sortimentsumfang von Verkaufsstellen des Lebensmitteleinzelhandels und kommen zum selben Ergebnis. 
Sehr uneinheitlich sind demgegenüber die Erkenntnisse zu Agglomerations- bzw. Wettbewerbseffekten durch die räumliche Nähe zu Mitbewerben derselben (intraformaler Wettbewerb) oder einer anderen Betriebsform (interformaler Wettbewerb). Ausgehend von der Annahme, dass hierdurch die Möglichkeit für Kopplungs- und Vergleichskäufe eröffnet wird, zeigt Wieland (2015: 180-182), dass die Nähe zu branchengleichen Anbietern unterschiedlicher Betriebsform (z. B. Verbrauchermarkt und Lebensmitteldiscounter) die Kundenzuflüsse des jeweiligen Marktes signifikant erhöht. Die räumliche Nähe zu Wettbewerbern derselben Betriebsform wird aber als reiner Konkurrenzeffekt, das heißt mit einer negativen Wirkung auf den eigenen Kundenzufluss, identifiziert (Tihi/Oruc 2012: 44-45; Wieland 2015: 180-182). Wird nicht zwischen Betriebsformen differenziert, finden Popkowski Leszczyc, Sinha und Sahgal (2004: 92-95) einen positiven Effekt nur für eine bestimmte Kundengruppe: service seeker.

Der Einfluss der Preispolitik ist schwerer zu quantifizieren: Sowohl Stated-choice-Befragungsexperimente als auch die Analyse realer Einkaufsentscheidungen zeigen, dass das durchschnittliche Preisniveau von Lebensmittelmärkten (Preis eines standardisierten Warenkorbs) eine negative Wirkung auf den Kundenzufluss hat (Lademann 2007: 153-155; Briesch/Chintagunta/Fox 2009: 183-186; Wieland 2015: 214-219). Allerdings ist die Berücksichtigung von Preisniveaus nicht trivial: Einerseits reagieren die Lebensmitteleinzelhandels-Filialisten schnell auf Preisanpassungen ihrer Mitbewerber, wobei sich deren Preiskonzepte deutlich angenähert haben, sodass der Unterschied zwischen den vormals sehr unterschiedlichen Ketten zumindest hinsichtlich des Preiseinstiegsbereiches schwindet (Lademann 2013: 16-17). Andererseits liegen nur in Ausnahmefällen aktuelle Daten zu standardisierten Warenkörben vor, die allerdings nie alle marktrelevanten Ketten umfassen und aufgrund o. g. Preisanpassungen nur eine Momentaufnahme darstellen können. Um kettenspezifische Einflüsse zu erfassen, können in modellgestützten Studien stattdessen Dummyvariablen für die jeweiligen Filialunternehmen als erklärende Variablen integriert werden. Wieland (2015: 214-219) zeigt, dass die Aussagekraft (Varianzaufklärung) eines solchen Modells deutlich höher ist als die eines Modells mit einem Preisindex als unabhängiger Variable.

\subsection{Einkaufsverhalten im Multi-Channel- Kontext}

Anders als in der o.g. explizit raumbezogenen Perspektive, deren Zielgröße die Wahl des Einkaufsstandortes ist, erfolgt die Untersuchung des Einkaufsverhaltens im Multi-Chan$n e l$-Kontext in der Regel auf der Ebene der Kanalwahl; das heißt es wird geprüft, welche angebots- und/oder nachfrageseitigen Attribute die Auswahl oder Nutzungsintensität eines spezifischen Kaufkanals (z. B. Onlinehandel) in Relation zu einem anderen Kaufkanal (z. B. stationärer Einzelhandel) erklären. Als methodischer Zugang fungieren hierbei quantitative Kanalwahlmodelle (z. B. binäres Logit-Modell für die Entscheidung für oder gegen einen Onlinekauf).

Viele verkehrswissenschaftliche Arbeiten erklären diese Wahl mit kanalspezifischen Transaktionskosten, die sich auf die Raumüberwindung entweder der zu erwerbenden Güter oder der Konsumentinnen/Konsumenten selbst beziehen: In einem Stated-choice-Befragungsexperiment analysiert Hsiao (2009: 90-93) die Rolle von Fahrtkosten, Fahrtzeit und Versandzeit bei der Auswahl des Kaufkanals (stationärer Handel vs. Onlineshop). Im Ergebnis senken Fahrtkosten und Fahrtzeit zum Laden die Wahrscheinlichkeit eines dortigen Kaufs, während eine steigende Versandzeit die Kunden tendenziell von einem Onlinekauf abhält. Anhand von Echtdaten der Kundschaft eines Multi-Channel-Lebensmittelhändlers untersuchen Chintagunta, Chu und Cebollada (2012: 103-111) die Einflüsse verschiedener Transaktionskostenformen auf die Wahrscheinlichkeit des Onlinekaufs. Hierbei zeigt sich, dass diese mit zunehmender Entfernung zum stationären Angebot und längerer Einkaufszeit steigt. Unsicherheitskosten durch eine mangelnde vorherige Produktbegutachtung senken aber die Wahrscheinlichkeit eines Onlinekaufs. Diese Ansätze erklären das Einkaufsverhalten im Wesentlichen anhand der konsumentenseitigen Abwägung verschiedener Formen von Transaktionskosten des Einkaufs unter der (impliziten) Annahme, dass die Kanalwahl als kostenminimierende Entscheidung erfolgt, wobei der zugrunde gelegte ,Kosten'-Begriff keinesfalls nur monetäre Aufwände umfasst. Prinzipiell sind diese Überlegungen anschlussfähig an die traditionellen Standorttheorien des Einzelhandels, in denen den Transportkosten der Nachfrager eine entscheidende Rolle zukommt (Mensing/ Neiberger 2018: 4-5).

Häufig werden objektive Attribute von Konsumentinnen und Konsumenten zur Erklärung der Kanalwahl herangezogen, z. B. demographische und ökonomische Eigenschaften sowie der Wohnort(typ). Im Hinblick auf räumliche Unterschiede im Einkaufsverhalten ist die Literaturlage nicht eindeutig: Viele Studien betrachten Onlinekäufe im Allgemeinen (das heißt unter Einschluss aller oder mehrerer Warengruppen) und finden diesbezüglich eine höhere Online-Affinität in urbanen Gebieten (z. B. Cao/Chen/ Choo 2013: 23-24; Mensing/Neiberger 2016: 117-122; Beckers/Cárdenas/Verhetsel 2018: 36-38). Zhen, Du, Cao et al. (2018: 296-297) betrachten Buch- und Bekleidungskäufe separat und bestätigen dieses Ergebnis, während Clarke, Thompson und Birkin (2015: 383-386) für Lebensmittelkäufe den umgekehrten Effekt finden. Allerdings 
überdeckt die Differenzierung nach ,städtischen“ und ,ländlichen' Gebieten die Einflüsse soziodemographischer und ökonomischer Konfigurationen: Als besonders ,online-affin " wurden beispielsweise immer wieder jüngere (und männliche) Konsumenten sowie solche mit einem höheren Bildungsgrad bzw. Einkommen identifiziert. Diese Tendenz erweist sich als unabhängig vom Raumtyp, jedoch sind besagte Gruppen (mit Ausnahme des Geschlechterunterschiedes) in urbanen Räumen stärker repräsentiert (Clarke/Thompson/Birkin 2015: 377-386; Mensing/Neiberger 2016: 122-123; Beckers/Cárdenas/Verhetsel 2018: 35-39; Wiegandt/Baumgart/Hangebruch et al. 2018: 257-258). Zudem wird häufig die Erreichbarkeit des stationären Einzelhandels als Determinante der Kanalwahl identifiziert: In vielen Studien wird bestätigt, dass die Wahrscheinlichkeit des Onlinekaufs mit steigender Entfernung vom Kundenwohnort zum Geschäft steigt, z. B. bei Lebensmittelkäufen (Clarke/Thompson/Birkin 2015: 386-387) und auch in Bezug auf generelles Online-Kaufverhalten (Ren/Kwan 2009: 270-275). Zhen, Du, Cao et al. (2018: 296-297) finden diesen Zusammenhang für Buchkäufe, jedoch nicht für Bekleidungskäufe. Demnach scheint der Raumtyp nicht die kausale Erklärungsgröße zu sein, sondern die Nähe zum stationären Einzelhandelsangebot, die im städtischen Raum tendenziell (aber nicht notwendigerweise) besser ausgeprägt ist. Die bisherigen Ergebnisse sprechen dafür, dass es im Hinblick auf den Aspekt der Erreichbarkeit deutliche Unterschiede zwischen den Warengruppen gibt. Beim Lebensmitteleinkauf im Multi-Channel-Kontext scheint, wie Clarke, Thompson und Birkin (2015: 386-387) zeigen, ein diesbezüglicher Zusammenhang zu bestehen.

In der Marketingforschung wird das Kanalwahlverhalten (auch) durch psychographische Charakteristika der Konsumentinnen und Konsumenten erklärt; hierzu zählen individuelle Einstellungen sowie Lebensstile. Diese Attribute werden in der Regel erst durch einzelne Befragungs-Items erfasst und später zu latenten Variablen aggregiert (z. B. durch Faktoranalyse); in einigen Fällen erfolgt auch eine Kundentypisierung (z. B. durch Clusteranalyse). Typische Einstellungsmuster, die mit einer größeren individuellen Neigung zum Onlinekauf einhergehen, sind unter anderem Offenheit gegenüber Technologie im Allgemeinen, Preissensibilität sowie eine Bequemlichkeits- oder Unabhängigkeitsorientierung (Ganesh/Reynolds/Luckett et al. 2010: 109-113; Schröder/Witek 2010: 92-97; Burkolter/Kluge 2011: 2426; Zaharia/Hackstetter 2017: 49-64; Zhai/Cao/Mokhtarian et al. 2017: 889-901). Einstellungen, die gegen die Wahl des Onlinekanals sprechen, sind unter anderem eine generelle Ablehnung des Kanals als solches, Serviceorientierung sowie Risikoaversion, z. B. hinsichtlich Datenschutz, Internetbetrug oder dem Risiko, das falsche Produkt geliefert zu bekommen (Schröder/Witek 2010: 92-97; Bezes
2016: 285-293; Zaharia/Hackstetter 2017: 49-64). In der Untersuchung von Wiegandt, Baumgart, Hangebruch et al. (2018: 259) werden Lebensstilgruppen berücksichtigt. Hierbei zeigt sich, dass Hedonisten und Unterhaltungssuchende signifikant mehr online einkaufen, wohingegen konservativbzw. liberal-gehobene Milieus eher dem stationären Handel zugewandt sind.

Eine besondere Rolle kommt der experimentellen Studie von Schmid und Axhausen (2019) zu, da hier alle oben genannten Aspekte in einem Kanalwahlmodell für zwei Sortimentsbereiche (Lebensmittel und Elektronik) berücksichtigt wurden. In ihrem Modell stellt eine latente Variable die individuelle ,Online-Affinität ' der Befragten dar, die multidimensional aus verschiedenen Einstellungs-Items gebildet wurde (unter anderem hinsichtlich Risikoaversion). Diese hat einen starken, positiven Einfluss auf die Auswahlwahrscheinlichkeit des Online-Kaufkanals. Gleichzeitig zeigt sich, dass online-affine Kunden deutlich preissensibler sind. Weiterhin werden drei Formen von kanalspezifischen Transaktionskosten ins Modell aufgenommen (Fahrtzeit zum stationären Anbieter, Lieferkosten und Lieferzeit bei der Online-Alternative), die alle einen negativen Effekt auf die Auswahl des jeweiligen Kaufkanals zeigen (Schmid/ Axhausen 2019: 163-169). Es muss aber bedacht werden, dass diesen Ergebnissen ein Stated-choice-Befragungsexperiment (das sind Entscheidungen zwischen fiktiven Alternativen) zugrunde liegt, das zudem unter der gedanklichen Prämisse erfolgte, dass keine Pkw zur privaten Nutzung zur Verfügung stehen. Eine Übertragbarkeit auf reales raumrelevantes Verhalten erscheint daher zumindest fraglich.

Ein grundsätzliches Problem aller genannten Ansätze besteht darin, dass sie auf die Kanalwahl abzielen, das heißt, viele zum Teil sehr heterogene Anbieter auf der Ebene der Kaufkanäle aggregieren. Es fehlt jedoch die Betrachtung des Einkaufsverhaltens auf der Ebene einzelner Anbieter in Form eines Modells der Einkaufsstättenwahl (Suel/Polak 2018: 590-591). Die genannten Ansätze beziehen sich zudem allesamt auf die Kanalwahl, wobei Online- und stationärer Handel als Gegensätze wahrgenommen werden. Inwiefern sich die Option von click and collect auf das Einkaufsverhalten auswirkt, ist - trotz der hohen Relevanz von cross-channel shopping (Boniversum 2018: 3-7) - bisher nicht berücksichtigt worden.

\section{Methodisches Vorgehen}

\subsection{Modellansatz}

Um die wichtigsten Determinanten des räumlichen Einkaufsverhaltens im Lebensmitteleinzelhandel empirisch zu ermitteln, wird ein mikroökonometrisches Modell der 
Einkaufsstättenwahl auf der Basis eines Hurdle-Modells verwendet. Dieser Modelltyp besteht aus zwei Gleichungen: Der erste Teil ist ein binäres Wahlmodell, dessen Zielgröße die Wahrscheinlichkeit ist, dass ein Individuum eine Entscheidung (hier: Einkauf bei einem bestimmten Anbieter) trifft (sogenannte participation equation). Die zweite Gleichung (sogenannte intensity equation) berücksichtigt nur die Fälle, in denen die bestimmte Entscheidung getroffen wurde (hier: dass bei einem bestimmten Anbieter eingekauft wird) und hat die daraus folgende Intensität (z. B. Häufigkeit, Menge) als Zielgröße (Cameron/Trivedi 2005: 680-681; Crowley/Eakins/ Jordan 2012: 205-206). Der mathematische Zweck des Modellansatzes ist die Berücksichtigung abhängiger Variablen mit einer sehr schiefen Verteilung, z. B. aufgrund einer fest definierten und sehr häufig vorkommenden Untergrenze (Greene 2012: 861-866).

Die Interpretation des Hurdle-Modells als Modell der Einkaufsstättenwahl folgt der Darstellung bei Wieland (2018: 376-378). Wie in einem Logit-Modell zur Einkaufsstättenwahl (Popkowski Leszczyc/Sinha/Sahgal 2004: 8990) wird der Nutzen der Auswahlalternative $j$ für die Entscheidungsperson $i$ in einer Nutzenfunktion ausgedrückt:

$U_{i j}=V_{i j}+\varepsilon_{i j}$

Dabei ist $U_{i j}$ der Nutzen der Alternative $j$ für Nachfrager $i, V_{i j}$ der durch das Modell erklärte Teil des Nutzens (sogenannter Repräsentativer Nutzen) und $\varepsilon_{i j}$ der Fehlerterm.

Als abhängige Variable fungieren im vorliegenden Fall empirisch ermittelte Einkaufsausgaben von Individuum $i$ bei Anbieter $j, S_{i j}$. Der erste Teil des Hurdle-Modells beinhaltet also die Entscheidung, $o b$ Individuum $i$ bei Anbieter $j$ einkauft, das heißt ob die dort getätigten Ausgaben $\left(S_{i j}\right)$ größer null sind. Er wird hier als binäres Logit-Modell formuliert, dessen Zielgröße die Wahrscheinlichkeit ist, dass Individuum $i$ bei Anbieter $j$ einkauft in Abhängigkeit des (repräsentativen) Nutzens des Anbieters $j$ für Individuum $i$ :

$\operatorname{Pr}\left[S_{i j}>0 \mid V_{i j}\right]=\frac{e^{V_{i j}}}{1+e^{V_{i j}}}$

Der repräsentative Nutzen steht in einem linearen Zusammenhang mit dem Logit (logarithmierte Chance des Eintretens):

$\ln \frac{\operatorname{Pr}\left[S_{i j}>0 \mid V_{i j}\right]}{1-\operatorname{Pr}\left[S_{i j}>0 \mid V_{i j}\right]}=V_{i j}$

In den zweiten Modellteil, der die Wie-Entscheidung beinhaltet, gehen nur die Fälle ein, bei denen tatsächlich ein Einkauf getätigt wurde $\left(S_{i j}>0\right)$. Im vorliegenden Fall wird dafür ein (zensiertes) Poisson-Zähldatenmodell verwendet, das den Erwartungswert der Ausgaben von Individuum $i$ bei Anbieter $j$ in Abhängigkeit des Nutzens modelliert:

$E\left(S_{i j}, S_{i j}>0 \mid V_{i j}\right)=\frac{\lambda_{i j}}{1-e^{-\lambda_{i j}}}$

Hierbei ist $\lambda_{i j}$ der Erwartungswert der Poisson-Verteilung, dessen natürlicher Logarithmus dem repräsentativen Nutzen entspricht:

$\ln \lambda_{i j}=V_{i j}$

Der Erwartungswert des gesamten Hurdle-Modells ist das Produkt der Auswahlwahrscheinlichkeit und des Erwartungswertes der intensity equation:

$$
\begin{aligned}
& E\left(S_{i j} \mid V_{i j}\right)= \\
& \quad\left(\operatorname{Pr}\left[S_{i j}>0 \mid V_{i j}\right]\right)\left(E\left[S_{i j}, S_{i j}>0 \mid V_{i j}\right]\right)
\end{aligned}
$$

Es werden zwei Modellvarianten geschätzt. Die erste Variante (I) beinhaltet nur Einkäufe bei Lebensmittelmärkten. Es handelt sich also um eine Anwendung des Hurdle-Modells für das räumliche Einkaufsverhalten im stationären Lebensmitteleinzelhandel. Diese verfolgt den Zweck, einerseits die bereits bekannten Erklärungsgrößen (vgl. Kapitel 2.1) zu überprüfen (und somit gleichzeitig die Plausibilität des Modellansatzes zu testen) und andererseits den Effekt der Click-and-collect-Option auf den Kundenzufluss zu ermitteln. Daher werden folgende erklärende Variablen in die Nutzenfunktion aufgenommen:

- $A_{j}$ : Artikelzahl von Anbieter $j$ (Annahme einer positiven Wirkung);

- $t_{i j}$ : Fahrtzeit von Konsument $i$ zu Anbieter $j$ (Annahme einer negativen Wirkung);

- $C_{j}$ : Clusterbildung des Anbieters $j$ in Bezug auf Mitbewerber derselben Branche, aber anderer Betriebsform (Annahme einer positiven Wirkung). Hierbei wird der von Wieland (2015: 136-138) und Mensing (2018: 107-109) genutzte Konzentrationsindikator in leicht abgewandelter Form verwendet:

$$
C_{j}=\sum_{\substack{k=1 \\ k \neq j}}^{K} D_{F_{j k}} A_{k} d_{j k}^{-\varphi}
$$

Es wird die (Luftlinien-)Distanz zwischen Anbieter $j$ und allen $K(k=1, \ldots, K)$ Mitbewerbern der jeweils anderen Betriebsform (Vollsortimenter bzw. Discounter) mit deren jeweiligem Attraktivitätswert (Artikelzahl $A_{k}$ ) gewichtet und summiert. Die Distanz wird mit einem Exponenten gewich- 
tet, der - wie im Huff-Modell - den Distanzwiderstand bzw. den überlinear hemmenden Effekt von Entfernungen beim Einkauf ausdrückt (Huff 1962: 18-19), hier jedoch beim Weg zwischen den jeweiligen Anbietern. Da für diesen Koeffizienten kein empirischer Wert vorliegt, wird er, analog zu den genannten Arbeiten, auf $\varphi=2$ gesetzt, was einem vergleichsweise hohen Distanzwiderstand entspricht. Direkt benachbarte Anbieter fallen somit stark ins Gewicht, während weiter entfernt liegende Einkaufsstätten den Konzentrationswert nur wenig erhöhen (Wieland 2015: 137).

In Formel (7) zeigt eine Dummyvariable $\left(D_{F j k}\right)$ an, ob Anbieter $j$ und $k$ eine unterschiedliche Betriebsform aufweisen $\left(D_{F j k}=1\right)$ oder nicht $\left(D_{F j k}=0\right)$; hierdurch werden nur Betriebe der jeweils anderen Betriebsform berücksichtigt.

- $D_{C C j}$ : Dummyvariable, die anzeigt, ob Anbieter $j$ einen Click-and-collect-Einkauf ermöglicht $\left(D_{C C j}=1\right)$ oder nicht $\left(D_{C C j}=0\right)$. Hierbei wird erwartet, dass dieser Service die Attraktivität des jeweiligen Marktes steigert, also eine positive Wirkung im Hinblick auf die Kundenzuflüsse entfaltet.

- Um kettenspezifische Effekte (z. B. Preisniveau) zu erfassen (die im vorliegenden Fall nicht zum Untersuchungsgegenstand gehören), werden Dummyvariablen für die relevanten Filialisten des Lebensmitteleinzelhandels in die Modellgleichung integriert. Die Variable $D_{g j}$ zeigt dabei an, ob Markt $j$ zu Kette $g$ gehört $\left(D_{g j}=1\right)$ oder nicht $\left(D_{g j}=0\right)$. Um eine Vergleichbarkeit zwischen den Untersuchungsgebieten zu gewährleisten und das Modell nicht zu überfrachten, wurden nur die Ketten $(g=1, \ldots, G)$ berücksichtigt, die in beiden Untersuchungsgebieten (vgl. Kapitel 3.2.1) mindestens zwei Filialstandorte haben.

- Bio-Lebensmittelmärkte, die hinsichtlich ihrer Sortimentsbreite konventionellen Supermärkten gleichzusetzen sind (z. B. Alnatura), werden in der Modellanalyse berücksichtigt. Allerdings besteht bei BioProdukten ein deutlich höheres Preisniveau als bei konventionellen Produkten (Haubach/Held 2015: 50-53). Daher ist zu erwarten, dass die Ausgaben je Einkauf - die die abhängige Variable im Modell darstellen unter ansonsten gleichen Bedingungen in Biomärkten höher sind als in anderen Betriebsformen des Lebensmitteleinzelhandels. Die Variable $D_{\text {Bioj }}$ zeigt daher an, ob es sich bei Anbieter $j$ um einen Bio-Supermarkt handelt (1) oder nicht (0).

- Da durchschnittliche Einkaufsbons stark schwanken können und auch gewisse Kaufkraftunterschiede besteh- en dürften, wird als letzte Kontrollvariable die gesamte erfasste Ausgabensumme von Nachfrager $i$ (d. h. über alle $n$ Anbieter) ins Modell aufgenommen:

$$
S_{i}=\sum_{j=1}^{n} S_{i j}
$$

Um einen anzunehmenden nichtlinearen Effekt der stetigen Variablen (vgl. Kapitel 2.1) erfassen und gleichzeitig die Regressionskoeffizienten als Elastizitäten interpretieren zu können (Wooldridge 2002: 647-648; Cameron/ Trivedi 2005: 470), werden die Variablen Artikelzahl, Fahrtzeit und Standortkonzentration in der ersten Modellvariante mit dem natürlichen Logarithmus (ln) transformiert. Der repräsentative Nutzen in Modellvariante I hat also folgende Gleichung:

$$
\begin{aligned}
V_{i j}^{I}= & \beta_{0}+\beta_{1} \ln A_{j}+\beta_{2} \ln t_{i j}+\beta_{3} \ln C_{j}+\beta_{4} D_{C C_{j}} \\
& +\beta_{5} D_{B_{i o_{j}}}+\sum_{g}^{G} \beta_{g} D_{g_{j}}+\beta_{15} \ln S_{i}
\end{aligned}
$$

In der zweiten Variante werden auch die relevanten Online-Lebensmittelanbieter berücksichtigt. Dieses Modell beschreibt also das Einkaufsverhalten im Lebensmitteleinzelhandel unter der Bedingung, dass stationäre Anbieter und Onlineshops gleichermaßen zur Verfügung stehen. In dieses Modell werden daher zusätzlich noch folgende Variablen aufgenommen:

- $s c_{j}$ : Lieferkosten von (Online-)Anbieter $j$ (Annahme einer negativen Wirkung)

- Analog zur Arbeit von Schmid und Axhausen (2019) werden eine Einstellungsvariable sowie - zwecks statistischer Kontrolle - demographische Faktoren ins Modell integriert (vgl. Kapitel 2.2). Die Online-Affinität von Individuum $i$ wird dabei über eine latente Variable (LV) dargestellt, die, analog zur genannten Arbeit, aus mehreren Items abgeleitet wird (vgl. Kapitel 3.2.2). Weiterhin wird eine Dummyvariable aufgenommen $\left(v s_{j}\right)$, die anzeigt, ob Anbieter $j$ ein stationärer Lebensmittelmarkt $\left(v s_{j}=1\right)$ oder ein Online-Anbieter $\left(v s_{j}=0\right)$ ist. Aus dem Regressionskoeffizienten des Interaktionsterms beider Variablen $\left(v s_{j}^{*} L V_{i}\right)$ geht dann hervor, ob ein steigender Grad an Online-Affinität die Wahrscheinlichkeit des Kaufs bei einem stationären Anbieter senkt (negatives Vorzeichen) oder steigert (positives Vorzeichen). Der Zweck dieser Berücksichtigung ist, das Ergebnis aus dem 
kontrafaktischen Design der o. g. Studie anhand von realen Einkaufsentscheidungen zu überprüfen. Hierbei wird erwartet, dass die Neigung, einen physischen Lebensmittelmarkt aufzusuchen, mit steigender Online-Affinität sinkt (d.h. dass der Regressionskoeffizient negativ ist).

- Zwei demographische Dummyvariablen und ihre jeweiligen Interaktionen mit $v s_{j}$ werden ins Modell aufgenommen: $D 65_{i}$ zeigt an, ob Nachfrager $i$ in der Altersgruppe 65 Jahre oder älter $(1=$ ja, $0=$ nein $)$ ist. Das Geschlecht wird mit der Variable $D f_{i}$ erfasst $(1=$ weiblich, $0=$ männlich).

Da in der zweiten Modellvariante Online- und stationäre Anbieter berücksichtigt werden, erreichen einige Variablen notwendigerweise Werte gleich null (z. B. Fahrtzeit bei Onlineshops). Daher kann in dieser Variante keine ln-Transformation dieser Variablen vorgenommen werden. Der repräsentative Nutzen in Modellvariante II hat dementsprechend folgende Gleichung:

$$
\begin{aligned}
V_{i j}^{I I}= & \beta_{0}+\beta_{1} \ln A_{j}+\beta_{2} t_{i j}+\beta_{3} \ln C_{j}+\beta_{4} D_{C C_{j}} \\
& +\beta_{5} s c_{j}+\beta_{6} D_{B i o_{j}}+\sum_{g}^{G} \beta_{g} D_{g_{j}} \\
& +\beta_{16} v s_{j}+\beta_{17} L V_{i}+\beta_{18} D 65_{i}+\beta_{19} D f_{i} \\
& +\beta_{20}\left(v s_{j} \cdot L V_{i}\right)+\beta_{21}\left(v s_{j} \cdot D 65_{i}\right) \\
& +\beta_{22}\left(v s_{j} \cdot D f_{i}\right)+\beta_{23} \ln S_{i}
\end{aligned}
$$

\subsection{Datenerhebung}

\subsubsection{Untersuchungsgebiete}

Um die räumliche Heterogenität Deutschlands annähernd abzubilden, wurden zwei unterschiedliche Regionen als Untersuchungsgebiete ausgewählt, die effektiv Verflechtungsräume darstellen bzw. in der Raumordnung als zusammengehörige Regionen definiert sind und zugleich sämtliche BBSR-Kreis- und Gemeindetypen ${ }^{1}$ abdecken.

Zum einen wurde der (ehemalige) Regionalverband Südniedersachsen ausgewählt, bestehend aus den (ehemaligen) Landkreisen Göttingen, Osterode am Harz (beide fusioniert zum Landkreis Göttingen im Jahr 2016), Northeim und Holzminden. Ausgehend von der Kreisstruktur bis 2016 und den siedlungsstrukturellen Kreistypen des BBSR besteht diese Region (536.093 Einwohner; Stand: 31.12.2015) aus drei dünn besiedelten ländlichen Kreisen und einem städ-

\footnotetext{
1 Vgl. https://www.bbsr.bund.de/BBSR/DE/forschung/ raumbeobachtung/Raumabgrenzungen/deutschland/ kreise/siedlungsstrukturelle-kreistypen/kreistypen.html (05.01.2021).
}

tischen Kreis. Die BBSR-Gemeindetypen der 93 Gemeinden in diesem Gebiet verteilen sich wie folgt: eine Großstadt $(1,08 \%)$, sechs Mittelstädte $(6,45 \%)$, acht größere Kleinstädte (8,60\%), 33 kleinere Kleinstädte $(35,48 \%)$ und 45 Landgemeinden $(48,39 \%)$. Ausgehend von der BBSRKreis- und Gemeindetypisierung handelt sich hierbei also um ein weit überwiegend ländliches Gebiet.

Zum anderen wurde der Regionalverband Mittlerer Oberrhein (1.025.230 Einwohner; Stand: 31.12.2015) ausgewählt, bestehend aus den Stadtkreisen Karlsruhe und Baden-Baden sowie den Landkreisen Karlsruhe und Rastatt. Das Gebiet setzt sich zusammen aus einer Großstadt (Karlsruhe) und drei städtischen Kreisen. Die 57 Gemeinden entsprechen folgenden BBSR-Gemeindetypen: eine Großstadt (1,75\%), 19 Mittelstädte (33,33\%), 27 gröBere Kleinstädte $(47,37 \%)$, sieben kleinere Kleinstädte $(12,28 \%)$ und drei Landgemeinden $(5,26 \%)$. Dieses zweite Untersuchungsgebiet ist demnach überwiegend städtisch geprägt.

\subsubsection{Konsumentenbefragung}

Das Ziel der Erhebung war eine größtmögliche Repräsentativität im Hinblick auf sämtliche Bevölkerungsgruppen (einschließlich ,Online-Verweigerer' sowie Minderjährige ohne eigenen Hausstand). Daher wurde in den Untersuchungsgebieten im Zeitraum von März bis Juni 2019 eine schriftlich-postalische Konsumentenbefragung durchgeführt, wofür zufällig ausgewählte Adressen von den jeweiligen Einwohnermeldeämtern beschafft wurden. Als Grundgesamtheit wurden alle Personen mit amtlichem Erstwohnsitz in den Gebieten ab einem Alter von 15 Jahren definiert. Diese Abgrenzung erfolgte anhand der Überlegung, dass ab diesem Alter eine gewisse finanzielle Autonomie bzw. ein annährend eigenständiges Kaufverhalten angenommen werden kann (Burkolter/Kluge 2011: 22). Die zufällig ausgewählten Einwohnerinnen und Einwohner hatten alternativ zum schriftlichen Ausfüllen des Fragebogens die Möglichkeit, diesen online zu bearbeiten. Eine Verzerrung besteht hierdurch nicht, da die Kontaktaufnahme über den Postweg an die Wohnadresse erfolgte.

Der standardisierte Fragebogen bestand aus drei Abschnitten: Im ersten Abschnitt wurden tatsächliche Einkaufsentscheidungen im Hinblick auf vier Sortimente (Lebensmittel, Bekleidung, Elektroartikel, Möbel) abgefragt. Wie in der Pionierarbeit von Huff (1962) sowie jüngeren modellgestützten Untersuchungen zum räumlichen Einkaufsverhalten (Wieland 2015: 130; Steiger 2017: 206-207) wurden hierfür die letzten Einkäufe abgefragt, unabhängig davon, ob die jeweilige Einkaufsstättenwahl ,typisch ' gewesen ist oder nicht. Um das Erinnerungsvermögen der Befragten nicht zu überfordern, wurden jeweils die letzten drei Einkäufe (Name sowie bei stationären Anbietern auch 
Tabelle 1 Soziodemographische Angaben der Befragten nach Untersuchungsgebieten

\begin{tabular}{|c|c|c|c|c|c|c|c|}
\hline \multirow[t]{3}{*}{ Attribut } & & \multicolumn{3}{|c|}{ Südniedersachsen } & \multicolumn{3}{|c|}{ Mittlerer Oberrhein } \\
\hline & & \multicolumn{2}{|c|}{ Stichprobe 2019} & \multirow{2}{*}{$\begin{array}{l}\text { Grundgesamtheit } \\
2018^{*} \\
\%\end{array}$} & \multicolumn{2}{|c|}{ Stichprobe 2019} & \multirow{2}{*}{$\begin{array}{l}\text { Grundgesamtheit } \\
2018^{*} \\
\%\end{array}$} \\
\hline & & $n$ & $\%$ & & $n$ & $\%$ & \\
\hline \multirow[t]{3}{*}{ Geschlecht } & weiblich & 155 & 52,7 & 51,1 & 598 & 56,5 & 50,1 \\
\hline & männlich & 138 & 46,9 & 48,9 & 448 & 42,3 & 49,9 \\
\hline & keine Angabe & 1 & 0,3 & -- & 12 & 1,1 & -- \\
\hline \multirow[t]{6}{*}{ Altersgruppe } & $15-<18$ & 10 & 3,4 & 3,2 & 22 & 2,1 & 3,1 \\
\hline & $18-<25$ & 37 & 12,7 & 9,8 & 89 & 8,4 & 9,8 \\
\hline & $25-<45$ & 55 & 18,8 & 25,8 & 247 & 23,4 & 29,7 \\
\hline & $45-<65$ & 91 & 31,2 & 34,2 & 425 & 40,2 & 33,7 \\
\hline & $65-<75$ & 57 & 19,5 & 12,6 & 158 & 15,0 & 11,2 \\
\hline & $>=75$ & 42 & 14,4 & 14,5 & 115 & 10,9 & 12,6 \\
\hline \multirow[t]{5}{*}{ Haushaltsgröße } & 1 & 56 & 19,5 & k.A. & 165 & 15,7 & k.A. \\
\hline & 2 & 144 & 50,2 & k.A. & 478 & 45,5 & k.A. \\
\hline & 3 & 45 & 15,7 & k.A. & 190 & 18,1 & k.A. \\
\hline & 4 & 36 & 12,5 & k.A. & 155 & 14,7 & k.A. \\
\hline & $>4$ & 6 & 2,1 & k.A. & 63 & 6,0 & k.A. \\
\hline \multirow[t]{6}{*}{ Berufstätigkeit } & erwerbstätig & 131 & 45,2 & k.A. & 601 & 57,2 & k.A. \\
\hline & im Ruhestand & 100 & 34,5 & k.A. & 291 & 27,7 & k.A. \\
\hline & Schule/Studium & 41 & 14,1 & k.A. & 91 & 8,7 & k.A. \\
\hline & $\begin{array}{l}\text { nicht erwerbstätig } \\
\text { (Hausmann/-frau) }\end{array}$ & 6 & 2,1 & k.A. & 40 & 3,8 & k.A. \\
\hline & $\begin{array}{l}\text { nicht erwerbstätig } \\
\text { (arbeitssuchend) }\end{array}$ & 5 & 1,7 & k.A. & 7 & 0,7 & k.A. \\
\hline & Sonstiges & 7 & 2,4 & k.A. & 21 & 2,0 & k.A. \\
\hline
\end{tabular}

*Quelle: https://www.regionalstatistik.de/genesis/online/ (24.03.2020)

der Standort) sowie die beim jeweiligen Einkauf angefallene Ausgabensumme abgefragt.

Im zweiten Abschnitt des Fragebogens wurden 15 Items zur späteren Ableitung des psychographischen Konstruktes der Online-Affinität abgefragt. Hiervon entstammen neun Items der Arbeit von Schmid und Axhausen (2019: 159) und beziehen sich vor allem auf die Aspekte der Risikoaversion sowie weiterer Vor- und Nachteile des Onlinekaufs. Sechs weitere Items wurden hinzugefügt, wobei sich zwei auf ethische Aspekte des Onlinehandels bzw. die damit verbundenen gesellschaftlichen und Mediendebatten (vgl. Schaer 2018; Kläsgen 2019) beziehen und drei auf Aspekte des Datenschutzes im Internet. Entsprechend der Vorgehensweise bei Schmid und Axhausen (2019: 159-160) wurden mittels Faktorenanalyse zwei latente Variablen extrahiert, wovon eine das Konstrukt der Online-Affinität abbilden sollte. Im dritten Abschnitt des Fragebogens wurden demographische Informationen abgefragt.

In beiden Untersuchungsgebieten betrug die Bruttostichprobe 9.109 Personen (Südniedersachsen: 3.109; Mittlerer Oberrhein: 6.000). An der Befragung teilgenommen (Nettostichprobe) haben 1.375 Personen (Südniedersachsen: 297; Mittlerer Oberrhein: 1.078). Werden stichprobenneutrale
Ausfälle abgerechnet, lag die Rücklaufquote insgesamt bei 15,7\% (Südniedersachsen: 10,0\%; Mittlerer Oberrhein: $18,6 \%$ ). Die ungleichen Quoten dürften auf demographische Unterschiede und die unterschiedliche Bekanntheit der durchführenden Universität zurückzuführen sein. Tabelle 1 zeigt die soziodemographischen Eigenschaften der befragten Personen, aufgeschlüsselt nach Untersuchungsgebieten.

Tabelle 2 zeigt die 15 verwendeten Einstellungs-Items, deren Herkunft sowie ihre Zuordnung zu den beiden ermittelten latenten Variablen in der Faktorenanalyse. Diese Zuordnung entspricht im Wesentlichen den Ergebnissen von Schmid und Axhausen (2019: 159-160) und ist plausibel: Die Items 2 und 3, die auf eine generelle Risikoaversion bezüglich des Onlinekaufs abzielen, laden positiv auf den ersten Faktor, das heißt je geringer die Zustimmung zu den betreffenden Aussagen, desto höher der Faktorwert. Dasselbe Ergebnis zeigt sich bei anderen Items zu Aussagen, die sich auf Nachteile des Onlinekaufs beziehen (z. B. Items 4, 5 und 7). Je geringer die Zustimmung zu den Aussagen bezüglich möglicher ethischer Problemfelder des Onlinehandels (Items 9 und 10) und zu den Aussagen bezüglich der möglichen Datenschutzproblematik (Items 13-15), desto höher liegt der Faktorwert. Der erste Faktor repräsentiert 
Tabelle 2 Einstellungs-Items und zugehörige Faktoren

\begin{tabular}{|c|c|c|c|}
\hline Nr. & Einstellungs-Items ( 1 = trifft voll zu, ..., 4 = trifft nicht zu) & Faktor 1 & Faktor 2 \\
\hline $1^{*}$ & Ich bestelle häufig Produkte aus dem Internet. & & 0,59 \\
\hline $2^{*}$ & Einkaufen im Internet ist mit Risiken verbunden. & 0,61 & \\
\hline $3^{*}$ & $\begin{array}{l}\text { Betrug mit Bank- oder Kreditkartendaten ist einer der Gründe, warum ich das Einkaufen im Internet } \\
\text { nicht mag. }\end{array}$ & 0,68 & \\
\hline $4^{*}$ & Das Internet hat mehr Nachteile als Vorteile. & 0,57 & \\
\hline $5^{*}$ & $\begin{array}{l}\text { Ein Nachteil des Einkaufens im Internet ist, dass ich die Produkte vor dem Kauf nicht direkt selbst } \\
\text { begutachten kann. }\end{array}$ & 0,56 & \\
\hline $6^{*}$ & Das Einkaufen im Internet erleichtert den Vergleich von Preisen und Produkten. & & 0,59 \\
\hline 7* & $\begin{array}{l}\text { Das Risiko, ein falsches Produkt geliefert zu bekommen, ist einer der Gründe, warum ich das Einkau- } \\
\text { fen im Internet nicht mag. }\end{array}$ & 0,57 & \\
\hline 8 & $\begin{array}{l}\text { Egal, ob ich im Internet oder im Geschäft kaufe: Vor dem Kauf informiere ich mich häufig im Internet } \\
\text { über die Produkte und vergleiche Preise. }\end{array}$ & & 0,62 \\
\hline 9 & Das Einkaufen im Internet belastet die Umwelt, zum Beispiel durch den Zustelltransport. & 0,58 & \\
\hline 10 & Das Einkaufen im Internet fördert schlechte Arbeitsbedingungen, zum Beispiel für die Lieferanten. & 0,63 & \\
\hline $11^{*}$ & Für mich ist Einkaufen in der Regel eine lästige Pflicht. & & 0,59 \\
\hline $12^{*}$ & $\begin{array}{l}\text { Ich gehe gerne in Geschäfte, einfach nur um mich umzuschauen, auch dann, wenn ich gar nichts } \\
\text { kaufen will. }\end{array}$ & & $-0,52$ \\
\hline $13+$ & Ich habe das Gefühl, keine Kontrolle über meine Daten im Internet zu haben. & 0,64 & \\
\hline $14+$ & $\begin{array}{l}\text { Ich habe das Gefühl, dass meine persönlichen Daten innerhalb und außerhalb des Internets ausrei- } \\
\text { chend geschützt sind. }\end{array}$ & $-0,51$ & \\
\hline $15+$ & Der Schutz meiner persönlichen Daten ist mir generell sehr wichtig. & 0,36 & \\
\hline
\end{tabular}

Item übernommen aus: * Schmid/Axhausen (2019), + Sinus (2018)

dementsprechend - in stark vereinfachter Weise - die Online-Affinität in der Modellanalyse. Mit einem CronbachsAlpha-Wert von 0,80 ergibt sich eine annehmbare bis gute Reliabilität dieses Konstruktes.

\subsubsection{Erfassung der Anbieterdaten}

In die Untersuchung wurden alle Lebensmittelmärkte nach der Nielsen-Betriebsformendefinition in den beiden Untersuchungsgebieten aufgenommen, das heißt Super- und Verbrauchermärkte sowie Lebensmitteldiscounter (Nielsen 2016: 12-21). Die Erhebung der Standorte erfolgte im März 2019 anhand der Websites der Filialunternehmen (Marktsuche nach Postleitzahl bzw. Gemeinde). Für die Märkte wurden die Adresse, die Betriebsform und die Verfügbarkeit des Click-and-collect-Services erfasst. Über Anfragen bei den jeweiligen Regionalgesellschaften sowie öffentlich verfügbare oder durch lokale Planungsbehörden zur Verfügung gestellte Unterlagen wurden zudem die Verkaufsflächengrößen der Filialen zusammengetragen. Nach Abschluss der Befragung wurde die Liste der Anbieter um wenige Lebensmittelmärkte außerhalb des Untersuchungsgebietes (sofern sie aus mindestens zwei Gemeinden als Einkaufsquelle genannt wurden) und die relevanten OnlineAnbieter (sofern sie im Hinblick auf ihre Sortimentsbreite mit Lebensmittelmärkten vergleichbar sind) ergänzt.

Im vorliegenden Fall waren verkaufsstellenspezifische Artikelzahlen notwendig, um eine Vergleichbarkeit von stationären und Online-Anbietern herzustellen. Daher wurde die Artikelzahl wie folgt ermittelt: Für insgesamt 50 Super- und Verbrauchermärkte (v.a. Edeka, Rewe) lagen sowohl Artikelzahlen, die auf der jeweiligen Marktseite dokumentiert sind, als auch Verkaufsflächenangaben vor. Hieraus wurde ein kettenspezifischer Durchschnittswert (Artikel je qm Verkaufsfläche) gebildet und mit diesem für die übrigen Vollsortimenter die Artikelzahl geschätzt. Für die in ihrer Sortimentspolitik weitgehend standardisierten Discount-Filialisten lagen Daten zur durchschnittlichen Verkaufsfläche und Artikelzahl vor (Hahn Gruppe 2019: 31; Statista 2019), aus denen der o. g. Indikator berechnet und dementsprechend die Artikelzahl geschätzt wurde. Die Informationen zu den Online-Lebensmittelanbietern (Liefergebühr, Artikelzahl) konnte über ihre Websites abgerufen werden.

Um das Hurdle-Modell schätzen zu können, mussten die empirisch erhobenen Informationen weiterverarbeitet werden. Ähnlich wie bei anderen ökonometrischen Modellen der Einkaufsstättenwahl wurden die individuellen Ausgaben von Konsument $i(i=1, \ldots, m)$ bei Anbieter $j(j=1, \ldots, n)$ in eine Interaktionsmatrix mit $m^{*} n$ Einträgen transformiert (Wieland 2015: 133-139). Die Erfassung der Fahrtzeiten erfolgte anhand einer automatisierten Abfrage der OSRM (OpenStreetMap Routing Machine); hierbei wurde die kürzeste Pkw-Fahrtzeit ermittelt. 
Tabelle 3 Lebensmittelmärkte nach Betriebsformen und Untersuchungsgebieten

\begin{tabular}{|c|c|c|c|c|c|c|}
\hline \multirow[t]{2}{*}{ Betriebsform } & \multirow{2}{*}{$\begin{array}{l}\text { Märkte } \\
\text { [Anzahl] }\end{array}$} & \multicolumn{2}{|c|}{ Verkaufsfläche } & \multicolumn{2}{|l|}{ Artikelzahl } & \multirow{2}{*}{$\begin{array}{l}\text { Mit Click-and- } \\
\text { Collect-Angebot } \\
\text { [Anzahl] }\end{array}$} \\
\hline & & $\begin{array}{l}\text { Mittelwert } \\
\text { [qm] }\end{array}$ & $\begin{array}{l}\text { Standard- } \\
\text { abweichung } \\
\text { [qm] }\end{array}$ & $\begin{array}{l}\text { Mittelwert } \\
\text { [Anzahl] }\end{array}$ & $\begin{array}{l}\text { Standard- } \\
\text { abweichung } \\
\text { [Anzahl] }\end{array}$ & \\
\hline \multicolumn{7}{|c|}{ Untersuchungsgebiet 1 - Südniedersachsen } \\
\hline Supermärkte klein (< 400 qm) & 12 & 256,67 & 237,12 & $2.697,59$ & $1.651,32$ & 0 \\
\hline Supermärkte groß (400-1.000 qm) & 19 & 600,21 & 199,25 & $8.153,59$ & $4.357,20$ & 0 \\
\hline $\begin{array}{l}\text { Verbrauchermärkte klein } \\
(1.000-2.500 \mathrm{qm})\end{array}$ & 59 & $1.536,76$ & 416,45 & $18.612,25$ & $5.477,24$ & 5 \\
\hline $\begin{array}{l}\text { Verbrauchermärkte groß (> } 2.500 \\
\text { qm) }\end{array}$ & 19 & $4.248,32$ & $2.134,15$ & $40.021,71$ & $15.211,64$ & 0 \\
\hline Discounter & 88 & 856,08 & 199,74 & $4.961,81$ & $2.637,41$ & 0 \\
\hline Gesamt & 197 & $1.325,92$ & $1.247,22$ & $12.601,34$ & $12.369,35$ & 5 \\
\hline \multicolumn{7}{|c|}{ Untersuchungsgebiet 2 - Mittlerer Oberrhein } \\
\hline Supermärkte klein (< 400 qm) & 17 & 287,65 & 104,73 & $3.300,55$ & $1.201,74$ & 0 \\
\hline Supermärkte groß (400-1.000 qm) & 50 & 699,82 & 178,48 & $8.869,56$ & $3.703,07$ & 0 \\
\hline $\begin{array}{l}\text { Verbrauchermärkte klein } \\
(1.000-2.500 \text { qm) }\end{array}$ & 68 & $1.427,19$ & 332,06 & $18.181,87$ & $4.268,47$ & 7 \\
\hline $\begin{array}{l}\text { Verbrauchermärkte groß (> } 2.500 \\
\text { qm) }\end{array}$ & 26 & $5.281,15$ & $2.596,76$ & $47.448,96$ & $21.436,82$ & 1 \\
\hline Discounter & 174 & 854,66 & 188,71 & $4.860,06$ & $1.033,02$ & 0 \\
\hline Lebensmittelabteilung Warenhaus & 1 & $2.030,00$ & -- & $23.292,82$ & -- & 0 \\
\hline Gesamt & 336 & $1.264,34$ & $1.413,13$ & $11.424,32$ & $13.329,67$ & 8 \\
\hline Online-Anbieter & 2 & -- & -- & $5.406,50$ & $6.129,91$ & -- \\
\hline
\end{tabular}

\section{Ergebnisse}

\subsection{Deskriptive Darstellung: Einkaufsverhalten nach Betriebsformen und Vertriebskanälen}

Tabelle 3 und Abbildung 1 zeigen die Lebensmittelmärkte nach Betriebsformen und Untersuchungsgebieten. Insgesamt sind 533 Lebensmittelmärkte (Südniedersachsen: 197; Mittlerer Oberrhein: 336) für die Analyse relevant; knapp die Hälfte hiervon (49,2\%) sind Lebensmitteldiscounter. Insgesamt boten zum Erhebungszeitpunkt 13 Verbrauchermärkte (Südniedersachsen: 5; Mittlerer Oberrhein: 8) den Click-and-collect-Service an. Im ersten Untersuchungsgebiet waren zum Erhebungszeitpunkt keine regionalen Online-Lieferservices verfügbar und auch sonst wurden - von wenigen Einkäufen bei Spezialanbietern abgesehen - keine Online-Lebensmittelkäufe registriert. Im zweiten Untersuchungsgebiet erwiesen sich der dort verfügbare Rewe-Lieferservice sowie Amazon als relevant.

In der Befragung wurden insgesamt 3.011 Einkäufe (Südniedersachsen: 637; Mittlerer Oberrhein: 2.374) in Lebensmittelmärkten bzw. damit vergleichbaren OnlineAnbietern im Gesamtwert von 137.826 Euro (Südniedersachsen: 25.069 Euro; Mittlerer Oberrhein: 112.757 Euro) erfasst (vgl. Tabelle 4). Ein Vergleich der jeweiligen
(Markt-)Anteile und der durchschnittlichen Ausgaben je Einkauf zeigt die Unterschiede zwischen den Betriebsformen bzw. Einkaufskanälen des Lebensmitteleinzelhandels auf, die auf betriebsformenabhängigen Einkaufsfrequenzen bzw. Typen von Einkäufen beruhen (HDE/IFH 2018: 15; Reutterer/Teller 2009: 699). So werden beispielsweise Supermärkte unter 1.000 qm Verkaufsfläche (wozu auch vereinzelte Märkte mit dem City-Konzept gehören, z. B. Rewe City, Edeka xpress) tendenziell für kleinere Einkäufe aufgesucht, während große Verbrauchermärkte (ab 2.500 qm Verkaufsfläche, z. B. Real) die höchsten Durchschnittsausgaben aufweisen bzw. ihr Anteil an den Ausgaben deutlich höher liegt als bei den Einkäufen. In diesen Märkten werden tendenziell weniger häufige Großeinkäufe erledigt. Lebensmitteldiscounter werden zwar im zweiten Untersuchungsgebiet häufiger aufgesucht, sind allerdings dort auch im Hinblick auf Anzahl an Verkaufsstellen präsenter (vgl. Tabelle 3), ihr Ausgabenanteil liegt jedoch in beiden Gebieten bei etwa $35 \%$.

Nur im zweiten Untersuchungsgebiet wurden Online-Lebensmitteleinkäufe im Sinne der hier angesetzten Anbieterdefinition registriert; diese Anbieter (Online-Lieferservice von Rewe sowie Amazon) vereinen 0,55\% der Einkäufe und 0,53\% der zugehörigen Ausgaben. Zwar wird der Online-Anteil im Food-Bereich für 2019 mit 1,4\% angegeben (HDE/IFH 2020: 8), jedoch schließt dieser Anteil auch Spe- 


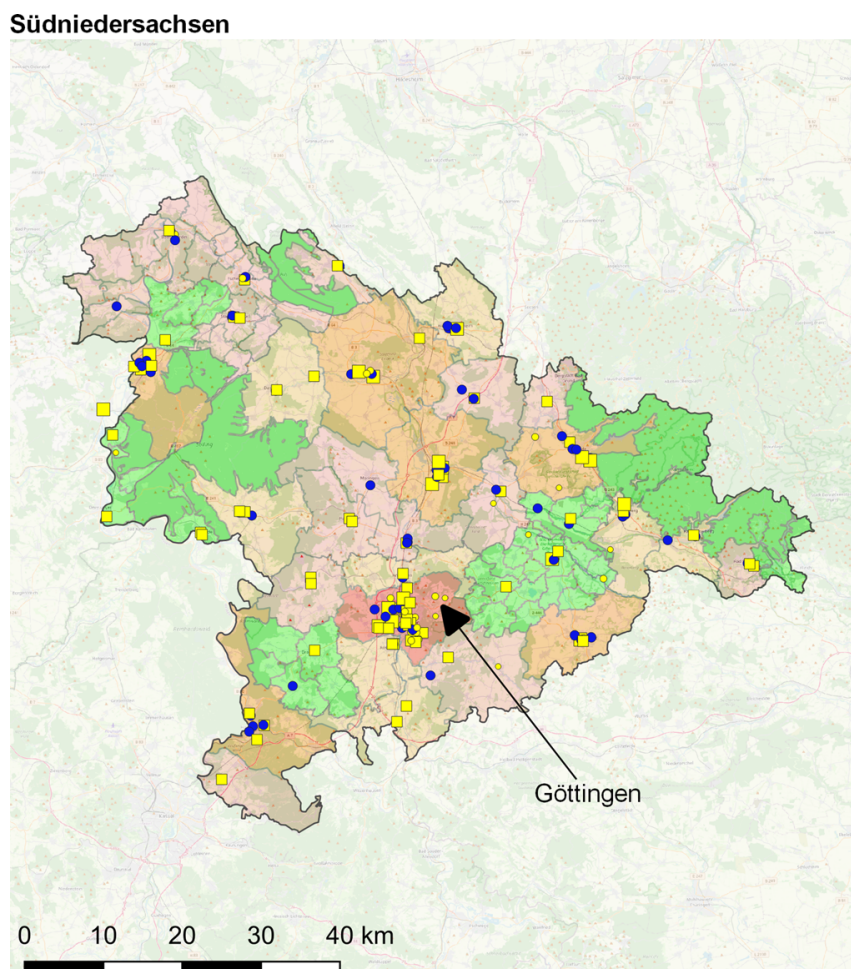

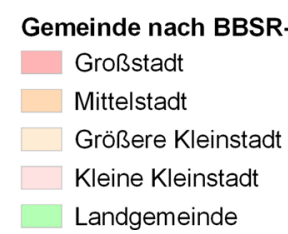

Supermarkt klein (bis 400 qm)

Supermarkt groß (400-999 qm)

Verbrauchermarkt klein (1.000-2.500 qm)

Verbrauchermarkt groß (> $2.500 \mathrm{qm})$

- LM-Discounter

((c) GeoBasis-DE / BKG 2015), OpenStreetMap

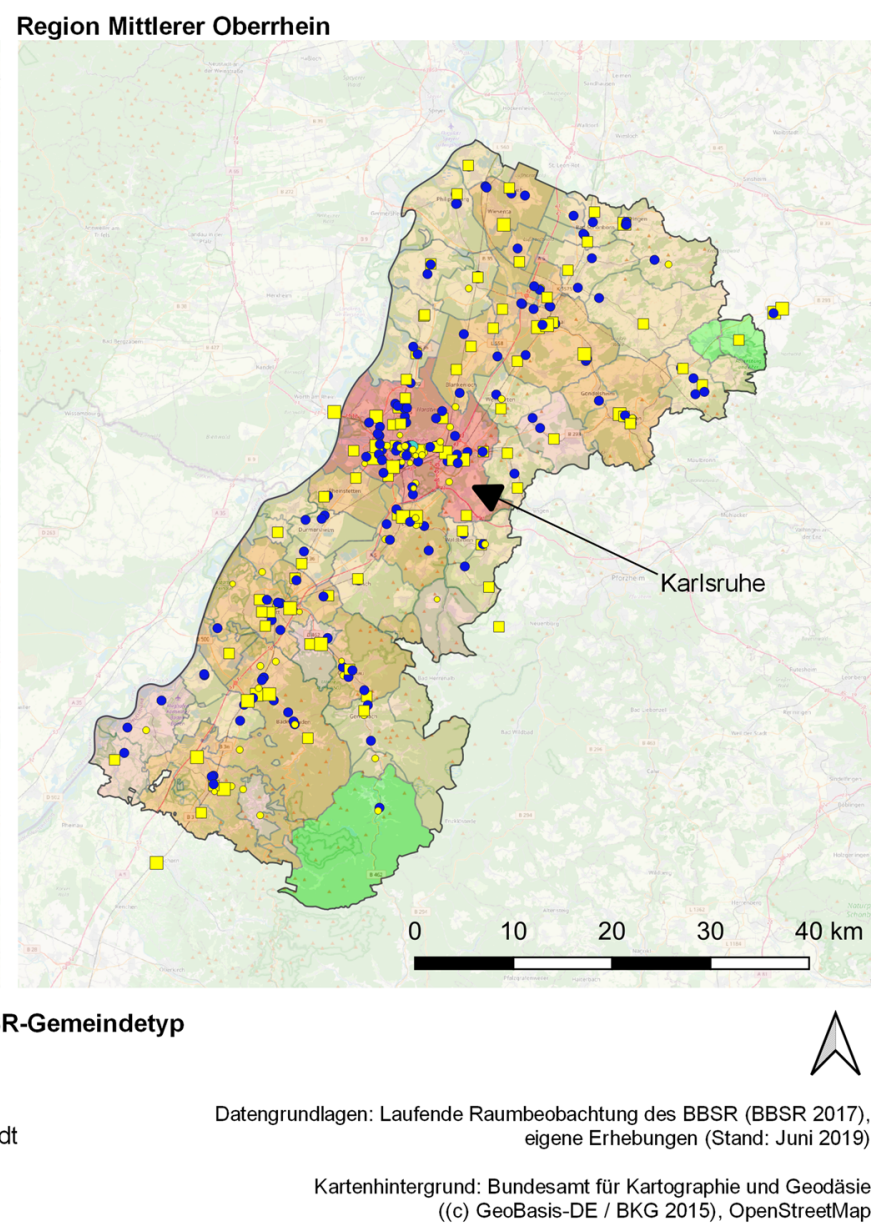

Abbildung 1 Lebensmittelmärkte nach Betriebsformen und Untersuchungsgebieten

zialanbieter (z. B. Spezialversände für Feinkost) mit ein, die in der hiesigen Betrachtung ausgeschlossen wurden. Insgesamt zeigt die deskriptive Darstellung bereits sehr große Unterschiede in den Einkaufshäufigkeiten und durchschnittlichen Ausgaben zwischen den Betriebsformen bzw. Vertriebskanälen auf, was die Sinnhaftigkeit des hier verwendeten Modellansatzes, der zwischen einer Einkaufsstättenwahl und den zugehörigen Ausgaben differenziert, unterstreicht.

\subsection{Modellergebnisse}

\subsubsection{Stationäre Lebensmittelanbieter in beiden Untersuchungsgebieten}

Tabelle 5 und Tabelle 6 zeigen die Hurdle-Modellergebnisse für die stationären Lebensmittelanbieter in den beiden Untersuchungsgebieten. Die mittlere Spalte zeigt die Regressionskoeffizienten des binären Logit-Modells, das die $O b$ Entscheidung abbildet (d.h. die Wahrscheinlichkeit, dass Individuum $i$ Anbieter $j$ frequentiert). Die rechte Spalte beinhaltet die Regressionskoeffizienten des Zähldatenmodells, bei dem nur die Fälle eingehen, bei denen tatsächlich ein
Einkauf getätigt wurde $\left(S_{i j}>0\right)$. Um die Aussagekraft der Artikelzahl-Variable zusätzlich zu validieren, wurden für die stationären Anbieter alternativ jeweils Modelle mit der Verkaufsflächengröße anstatt der Artikelzahl geschätzt. Diese Modelle (hier nicht dargestellt) weisen hinsichtlich der Koeffizienten der hier interessierenden Variablen keine nennenswerten Unterschiede auf.

Zunächst zeigt sich in beiden Untersuchungsgebieten eine grundsätzliche Bestätigung des Einflusses der beiden als Basisgrößen identifizierten Erklärungsvariablen des Einkaufsverhaltens im Lebensmitteleinzelhandel: Die Artikelzahl hat in beiden Untersuchungsgebieten und beiden Gleichungen einen signifikant positiven Effekt auf die Einkaufsstättenwahl bzw. die im Markt getätigten Ausgaben. Für die Pkw-Fahrtzeit vom Kundenwohnort zum Markt ergibt sich demgegenüber in allen Modellteilen beider Untersuchungsgebiete ein signifikant negativer Effekt. Die Koeffizienten sind aufgrund der ln-Transformation als Elastizitäten $\mathrm{zu}$ interpretieren (vgl. Kapitel 3.1.2): Ausgehend vom ersten Modellteil, der im Wesentlichen mit dem Ergebnis von Discrete-choice-Modellen (vgl. Kapitel 2.1) vergleich- 
Tabelle 4 Erfasste Einkäufe und Ausgaben nach Betriebsformen und Untersuchungsgebieten

\begin{tabular}{|c|c|c|c|c|}
\hline \multirow[t]{2}{*}{ Betriebsform } & \multirow{2}{*}{$\begin{array}{l}\text { Einkäufe } \\
\text { Anteile } \\
{[\%]}\end{array}$} & \multicolumn{3}{|c|}{ Ausgaben } \\
\hline & & $\begin{array}{l}\text { Anteile } \\
\text { [\%] }\end{array}$ & $\begin{array}{l}\text { Mittelwert } \\
\text { [Euro] }\end{array}$ & $\begin{array}{l}\text { Standardabweichung } \\
\text { [Euro] }\end{array}$ \\
\hline \multicolumn{5}{|c|}{ Untersuchungsgebiet 1 - Südniedersachsen (Einkäufe: $\Sigma$ 637; Ausgaben: $\Sigma 25.069$ Euro) } \\
\hline Supermärkte $(<1.000$ qm) & 10,52 & 6,42 & 31,57 & 22,60 \\
\hline Verbrauchermärkte klein (1.000-2.500 qm) & 35,16 & 32,29 & 41,95 & 41,53 \\
\hline Verbrauchermärkte groß (> 2.500 qm) & 21,51 & 25,49 & 52,80 & 43,15 \\
\hline Discounter & 32,81 & 35,80 & 47,73 & 54,18 \\
\hline \multicolumn{5}{|c|}{ Untersuchungsgebiet 2 - Mittlerer Oberrhein (Einkäufe: $\Sigma$ 2.374; Ausgaben: $\Sigma 112.757$ Euro) } \\
\hline Supermärkte $(<1.000$ qm) & 13,61 & 11,81 & 48,79 & 52,20 \\
\hline Verbrauchermärkte klein (1.000-2.500 qm) & 26,33 & 26,84 & 56,68 & 59,86 \\
\hline Verbrauchermärkte groß (> 2.500 qm) & 19,38 & 25,50 & 72,60 & 69,20 \\
\hline Discounter & 39,76 & 35,00 & 45,42 & 36,19 \\
\hline Lebensmittelabteilung Warenhaus & 0,38 & 0,32 & 51,71 & 45,04 \\
\hline Online-Anbieter & 0,55 & 0,53 & 65,78 & 57,68 \\
\hline
\end{tabular}

bar ist, zeigt sich der Effekt der Artikelzahl im ersten Untersuchungsgebiet als unterproportional $(0,856)$, im zweiten Untersuchungsgebiet als proportional bzw. leicht überproportional (1,047). Umgekehrt ist der Effekt der Erreichbarkeit der Einkaufsstätte hochgradig überproportional: Eine 1-\%-Erhöhung der Fahrtzeit senkt die Auswahlchance um 2,708 bzw. 3,045 \%. In den jeweiligen Zähldatenmodellen wird ebenfalls ein positiver Effekt der Artikelzahl und ein negativer Effekt der Fahrtzeit gefunden. Unter ansonsten gleichen Bedingungen steigert also die Sortimentstiefe den Kaufkraftzufluss, während dieser mit steigender Fahrtzeit vom Kundenwohnort überproportional sinkt; diese Ergebnisse entsprechen vorherigen Studien sowie den mikrotheoretischen Annahmen des Huff-Modells (vgl. Kapitel 2.1).

Die zweite Standortvariable, die sich auf das Niveau der Clusterbildung mit Mitbewerbern anderer Betriebsformen bezieht, hat jeweils im Logit-Modell keinen signifikanten Einfluss, beeinflusst also die Einkaufsstättenwahl nicht. Betrachtet man allerdings beide Zähldatenmodelle, zeigt sich ein signifikant negativer Effekt auf die tatsächlichen Einkaufsausgaben. Dieses Ergebnis deutet darauf hin, dass - zumindest im hiesigen Fall - Wettbewerbseffekte mögliche positive Agglomerationseffekte durch die Möglichkeit von Kopplungs- und Vergleichskäufen überlagern.

Entgegen der Erwartungen kann für die bei bestimmten Super- und Verbrauchermärkten dargebotene Click-and-collect-Option kein signifikant positiver Effekt hinsichtlich der Einkaufsstättenwahl festgestellt werden: In beiden Untersuchungsgebieten ist der Koeffizient der Dummyvariablen, die die Verfügbarkeit dieser Form von Cross-Channel-Integration anzeigt, im Logit-Modell nicht signifikant. Super- und Verbrauchermärkte, die diesen Service anbieten, profitieren demnach im Hinblick auf die Einkaufswahrscheinlichkeit nicht davon. Allerdings zeigt sich im zweiten Untersu- chungsgebiet ein positiver Einfluss auf die Einkaufsausgaben. Abgesehen davon zeigt sich der Click-and-collect-Service demnach nicht als hervortretendes Attraktivitätsmerkmal von Verbrauchermärkten.

Die kettenspezifischen Effekte, die in der vorliegenden Untersuchung nur den Charakter von Kontrollvariablen haben, sind in beiden Untersuchungsgebieten tendenziell vergleichbar: So zeigt sich vor allem, dass der intrinsische Nutzen von Aldi übergreifend feststellbar ist, da der Koeffizient der zugehörigen Dummyvariablen in beiden Teilen beider Modelle positiv und signifikant ist. Das heißt, unter ansonsten konstanten Bedingungen erhöht die Marke Aldi die Auswahlchance sowie den Kaufkraftzufluss. Derselbe Effekt lässt sich für Lidl-Märkte aufzeigen, abgesehen davon, dass der Koeffizient im Logit-Modell in Südniedersachsen nicht signifikant ist. Diese Ketten erscheinen demnach als beliebter, was auch mit ihren weit überdurchschnittlichen Flächenproduktivitäten - im Vergleich der Filialisten kongruent ist (Hahn Gruppe 2019: 31). Der gegenteilige Effekt kann für das Label nahkauf festgestellt werden. Der positive Koeffizient für Biomärkte ist sehr plausibel, da aufgrund eines höheren Preisniveaus auch durchschnittlich höhere Einkaufsbons anzunehmen sind.

\subsubsection{Stationäre und Online-Lebensmittelanbieter in der Region Mittlerer Oberrhein}

Da nur im zweiten Untersuchungsgebiet überhaupt Einkäufe im Lebensmittel-Onlinehandel registriert wurden, ist die zweite Modellvariante auch nur für die Region Mittlerer Oberrhein anwendbar. Die Interpretation der Koeffizienten gegenüber der ersten Modellvariante ändert sich nur dahingehend, dass mehrere stetige Variablen nicht ln-transformiert sind und dementsprechend nicht mehr als Elastizitäten 
Tabelle 5 Hurdle-Modell für die Lebensmittelmärkte in Südniedersachsen

\begin{tabular}{|c|c|c|}
\hline $\begin{array}{l}\text { Unabhängige Varia- } \\
\text { blen }\end{array}$ & $\begin{array}{l}\text { Koeffizienten } \\
\text { Logit-Modell } \\
\text { (Standardfehler) }\end{array}$ & $\begin{array}{l}\text { Koeffizienten } \\
\text { Zähldatenmo- } \\
\text { dell (Standard- } \\
\text { fehler) }\end{array}$ \\
\hline In Artikelzahlj & $\begin{array}{l}0,856^{\star * *} \\
(0,110)\end{array}$ & $\begin{array}{l}0,214^{* * *} \\
(0,016)\end{array}$ \\
\hline In Pkw-Fahrtzeitij & $\begin{array}{l}-2,708^{* * *} \\
(0,066)\end{array}$ & $\begin{array}{l}-0,086^{* * *} \\
(0,010)\end{array}$ \\
\hline $\begin{array}{l}\text { In Standort- } \\
\text { konzentration }\end{array}$ & $\begin{array}{l}0,025 \\
(0,028)\end{array}$ & $\begin{array}{l}-0,012^{* * *} \\
(0,004)\end{array}$ \\
\hline $\begin{array}{l}\text { Dummy Click and } \\
\text { collect }_{j}\end{array}$ & $\begin{array}{l}-0,319 \\
(0,340)\end{array}$ & $\begin{array}{l}-0,033 \\
(0,049)\end{array}$ \\
\hline Dummy Aldij & $\begin{array}{l}1,054^{\star * *} \\
(0,305)\end{array}$ & $\begin{array}{l}0,448^{* * *} \\
(0,044)\end{array}$ \\
\hline Dummy Edekaj & $\begin{array}{l}-0,123 \\
(0,209)\end{array}$ & $\begin{array}{l}-0,070^{* * *} \\
(0,027)\end{array}$ \\
\hline Dummy Kauflandj & $\begin{array}{l}0,621^{* *} \\
(0,254)\end{array}$ & $\begin{array}{l}-0,072^{* *} \\
(0,032)\end{array}$ \\
\hline Dummy Lidlij & $\begin{array}{l}0,357 \\
(0,322)\end{array}$ & $\begin{array}{l}0,282^{* * *} \\
(0,046)\end{array}$ \\
\hline Dummy nahkaufj & $\begin{array}{l}-1,342^{* * *} \\
(0,450)\end{array}$ & $\begin{array}{l}-0,412^{* * *} \\
(0,077)\end{array}$ \\
\hline Dummy Nettoj & $\begin{array}{l}-0,168 \\
(0,305)\end{array}$ & $\begin{array}{l}-0,071 \\
(0,045)\end{array}$ \\
\hline Dummy Pennyj & $\begin{array}{l}-0,043 \\
(0,343)\end{array}$ & $\begin{array}{l}0,143^{* * *} \\
(0,053)\end{array}$ \\
\hline Dummy Realj & $\begin{array}{l}0,206 \\
(0,313)\end{array}$ & $\begin{array}{l}0,005 \\
(0,038)\end{array}$ \\
\hline Dummy Rewej & $\begin{array}{l}-0,047 \\
(0,211)\end{array}$ & $\begin{array}{l}-0,040 \\
(0,029)\end{array}$ \\
\hline Dummy Biomarkt ${ }_{j}$ & $\begin{array}{l}0,538 \\
(0,373)\end{array}$ & $\begin{array}{l}0,219 * * * \\
(0,060)\end{array}$ \\
\hline In Ausgaben & $\begin{array}{l}0,395^{\star * *} \\
(0,068)\end{array}$ & $\begin{array}{l}0,960^{* * *} \\
(0,010)\end{array}$ \\
\hline Konstante & $\begin{array}{l}-6,787 * * * \\
(1,124)\end{array}$ & $\begin{array}{l}-2,464^{\star * *} \\
(0,159)\end{array}$ \\
\hline Beobachtungen & \multicolumn{2}{|l|}{54.906} \\
\hline Log Likelihood & \multicolumn{2}{|l|}{$-6.406,995$} \\
\hline AIC & \multicolumn{2}{|l|}{$12.877,99$} \\
\hline
\end{tabular}

Anmerkung: *** $p<0,01 ;{ }^{* *} p<0,05 ;{ }^{*} p<0,1$

aufgefasst werden können; dies betrifft allerdings nicht die Richtung des Einflusses.

Der ,Attraktivitäts'-Indikator für die Anbieter beider Kaufkanäle (Artikelzahl) zeigt im Modell (Tabelle 7) eine positiv-unterlineare Wirkung. Das heißt, die bereits im Huff-Modell angenommene und in vielen Studien zur Einkaufsstättenwahl im stationären Handel festgestellte degressive Wirkung des Sortiments (abnehmender Grenznutzen; vgl. Kapitel 2.1) ist auch im direkten Vergleich von Offline- und Onlinehändlern zu bestätigen. Auch die Agglomerationsvariable und die Dummyvariable für die Click-and-collect-Verfügbarkeit zeigen im Multi-Channel-
Tabelle 6 Hurdle-Modell für die Lebensmittelmärkte in der Region Mittlerer Oberrhein

\begin{tabular}{|c|c|c|}
\hline $\begin{array}{l}\text { Unabhängige } \\
\text { Variablen }\end{array}$ & $\begin{array}{l}\text { Koeffizienten } \\
\text { Logit-Modell } \\
\text { (Standardfehler) }\end{array}$ & $\begin{array}{l}\text { Koeffizienten Zählda- } \\
\text { tenmodell (Standard- } \\
\text { fehler) }\end{array}$ \\
\hline In Artikelzahlj & $\begin{array}{l}1,047^{* * *} \\
(0,057)\end{array}$ & $\begin{array}{l}0,169^{* * *} \\
(0,007)\end{array}$ \\
\hline $\begin{array}{l}\text { In Pkw- } \\
\text { Fahrtzeitij }_{i j}\end{array}$ & $\begin{array}{l}-3,045^{* * *} \\
(0,035)\end{array}$ & $\begin{array}{l}-0,009^{* *} \\
(0,005)\end{array}$ \\
\hline $\begin{array}{l}\text { In Standort- } \\
\text { konzentrationj }\end{array}$ & $\begin{array}{l}0,016 \\
(0,014)\end{array}$ & $\begin{array}{l}-0,004^{* * *} \\
(0,001)\end{array}$ \\
\hline $\begin{array}{l}\text { Dummy Click } \\
\text { and collect; }\end{array}$ & $\begin{array}{l}0,037 \\
(0,148)\end{array}$ & $\begin{array}{l}0,060^{* * *} \\
(0,017)\end{array}$ \\
\hline Dummy Aldij & $\begin{array}{l}1,824^{\star * *} \\
(0,135)\end{array}$ & $\begin{array}{l}0,162^{* * *} \\
(0,017)\end{array}$ \\
\hline Dummy Edekaj & $\begin{array}{l}0,173 \\
(0,117)\end{array}$ & $\begin{array}{l}0,021^{*} \\
(0,012)\end{array}$ \\
\hline $\begin{array}{l}\text { Dummy } \\
\text { Kaufland }\end{array}$ & $\begin{array}{l}0,834^{\star \star *} \\
(0,161)\end{array}$ & $\begin{array}{l}0,133^{\star \star *} \\
(0,017)\end{array}$ \\
\hline Dummy Lidl ${ }_{j}$ & $\begin{array}{l}1,367^{\star * *} \\
(0,144)\end{array}$ & $\begin{array}{l}0,079 * * * \\
(0,019)\end{array}$ \\
\hline Dummy nahkaufj & $\begin{array}{l}-0,440^{*} \\
(0,230)\end{array}$ & $\begin{array}{l}0,046 \\
(0,030)\end{array}$ \\
\hline Dummy Nettoj & $\begin{array}{l}0,134 \\
(0,170)\end{array}$ & $\begin{array}{l}-0,126^{* \star *} \\
(0,023)\end{array}$ \\
\hline Dummy Pennyj & $\begin{array}{l}0,369 * * \\
(0,156)\end{array}$ & $\begin{array}{l}-0,060^{\star * *} \\
(0,021)\end{array}$ \\
\hline Dummy Realj & $\begin{array}{l}0,124 \\
(0,153)\end{array}$ & $\begin{array}{l}-0,039^{* *} \\
(0,016)\end{array}$ \\
\hline Dummy Rewe $j$ & $\begin{array}{l}0,441^{* * *} \\
(0,129)\end{array}$ & $\begin{array}{l}-0,046^{* * *} \\
(0,015)\end{array}$ \\
\hline $\begin{array}{l}\text { Dummy } \\
\text { Biomarkt; }\end{array}$ & $\begin{array}{l}0,667 * * * \\
(0,167)\end{array}$ & $\begin{array}{l}0,212^{\star * *} \\
(0,020)\end{array}$ \\
\hline In Ausgaben ${ }_{i}$ & $\begin{array}{l}0,244^{* * *} \\
(0,035)\end{array}$ & $\begin{array}{l}0,953^{* * *} \\
(0,005)\end{array}$ \\
\hline Konstante & $\begin{array}{l}-8,272^{* * *} \\
(0,609)\end{array}$ & $\begin{array}{l}-2,215^{* * *} \\
(0,073)\end{array}$ \\
\hline Beobachtungen & \multicolumn{2}{|c|}{322.156} \\
\hline Log Likelihood & \multicolumn{2}{|c|}{$-28.799,94$} \\
\hline AIC & \multicolumn{2}{|c|}{$57.663,89$} \\
\hline
\end{tabular}

Anmerkung: *** $p<0,01 ;{ }^{* *} p<0,05 ;{ }^{*} p<0,1$

Modell dieselben Wirkungen wie im Hurdle-Modell für die stationären Märkte in der Region Mittlerer Oberrhein.

Im Logit-Modell sind sowohl die Pkw-Fahrtzeit (bei stationären Anbietern) als auch die Liefergebühr (bei OnlineAnbietern) signifikant mit einem negativen Vorzeichen. Das heißt, eine Erhöhung der Fahrtzeit bzw. der Liefergebühr senkt die Auswahlchance des Anbieters. Dieses Ergebnis bestätigt anhand der Analyse realer Einkaufsentscheidungen die Erkenntnisse aus kontrafaktischen Befragungsexperimenten, in denen diese beiden Variablen als kanalspezifische Transaktionskosten aufgefasst werden (vgl. Kapitel 2.2). Im Zähldatenmodell hat die Liefergebühr allerdings 
Tabelle 7 Hurdle-Modell für die Lebensmittelmärkte und Lebensmittel-Online-Anbieter in der Region Mittlerer Oberrhein

\begin{tabular}{|c|c|c|}
\hline Unabhängige Variablen & $\begin{array}{l}\text { Koeffizienten } \\
\text { Logit-Modell } \\
\text { (Standardfehler) }\end{array}$ & $\begin{array}{l}\text { Koeffizienten } \\
\text { Zähldaten- } \\
\text { modell } \\
\text { (Standard- } \\
\text { fehler) }\end{array}$ \\
\hline In Artikelzahl j & $\begin{array}{l}0,928^{* * *} \\
(0,057)\end{array}$ & $\begin{array}{l}0,188^{* * *} \\
(0,007)\end{array}$ \\
\hline Pkw-Fahrtzeit ${ }_{i j}$ & $\begin{array}{l}-0,327^{* * *} \\
(0,005)\end{array}$ & $\begin{array}{l}-0,001^{* * *} \\
(0,001)\end{array}$ \\
\hline $\begin{array}{l}\text { In Standort- } \\
\text { konzentration }_{j}+0,001\end{array}$ & $\begin{array}{l}0,010 \\
(0,014)\end{array}$ & $\begin{array}{l}-0,006^{* * *} \\
(0,002)\end{array}$ \\
\hline Dummy Click and collect; & $\begin{array}{l}0,065 \\
(0,143)\end{array}$ & $\begin{array}{l}0,065^{* * *} \\
(0,018)\end{array}$ \\
\hline Liefergebühr $r_{j}$ & $\begin{array}{l}-2,069^{* * *} \\
(0,582)\end{array}$ & $\begin{array}{l}0,103 \\
(0,159)\end{array}$ \\
\hline Dummy Aldi ${ }_{j}$ & $\begin{array}{l}1,429 * * * \\
(0,128)\end{array}$ & $\begin{array}{l}0,214^{* * *} \\
(0,018)\end{array}$ \\
\hline Dummy Edekaj & $\begin{array}{l}0,067 \\
(0,113)\end{array}$ & $\begin{array}{l}0,049^{* * *} \\
(0,013)\end{array}$ \\
\hline Dummy Kauflandj & $\begin{array}{l}0,608^{* * *} \\
(0,163)\end{array}$ & $\begin{array}{l}0,168^{* * *} \\
(0,017)\end{array}$ \\
\hline Dummy Lidlj & $\begin{array}{l}1,016^{* * *} \\
(0,138)\end{array}$ & $\begin{array}{l}0,124^{* * *} \\
(0,019)\end{array}$ \\
\hline Dummy nahkaufj & $\begin{array}{l}-0,195 \\
(0,203)\end{array}$ & $\begin{array}{l}0,083^{* * *} \\
(0,031)\end{array}$ \\
\hline Dummy Nettoj & $\begin{array}{l}0,163 \\
(0,159)\end{array}$ & $\begin{array}{l}-0,077^{* * *} \\
(0,024)\end{array}$ \\
\hline Dummy Pennyj & $\begin{array}{l}0,250^{*} \\
(0,149)\end{array}$ & $\begin{array}{l}-0,034 \\
(0,022)\end{array}$ \\
\hline Dummy Realj & $\begin{array}{l}-0,028 \\
(0,153)\end{array}$ & $\begin{array}{l}-0,045^{* * *} \\
(0,016)\end{array}$ \\
\hline Dummy Rewe $_{j}$ & $\begin{array}{l}0,286^{* *} \\
(0,124)\end{array}$ & $\begin{array}{l}-0,031^{*} \\
(0,016)\end{array}$ \\
\hline Dummy Biomarktj & $\begin{array}{l}0,500^{* * *} \\
(0,156)\end{array}$ & $\begin{array}{l}0,278^{* * *} \\
(0,020)\end{array}$ \\
\hline Dummy stationär ${ }_{j}$ & $\begin{array}{l}-4,949^{*} \\
(2,649)\end{array}$ & $\begin{array}{l}0,767 \\
(0,574)\end{array}$ \\
\hline LV Online-Affinitäti & $\begin{array}{l}0,800^{* *} \\
(0,386)\end{array}$ & $\begin{array}{l}0,372^{* * *} \\
(0,098)\end{array}$ \\
\hline Dummy Alter $65+i$ & $\begin{array}{l}0,779 \\
(0,874)\end{array}$ & $\begin{array}{l}0,851^{* * *} \\
(0,256)\end{array}$ \\
\hline Dummy weiblichi & $\begin{array}{l}-0,054 \\
(0,829)\end{array}$ & $\begin{array}{l}0,112 \\
(0,240)\end{array}$ \\
\hline $\begin{array}{l}\text { Dummy stationär } r_{j} \text { * LV } \\
\text { Online-Affinität } t_{i}\end{array}$ & $\begin{array}{l}-0,771^{* *} \\
(0,387)\end{array}$ & $\begin{array}{l}-0,360^{* * *} \\
(0,098)\end{array}$ \\
\hline $\begin{array}{l}\text { Dummy stationär }{ }_{j} \text { * } \\
\text { Dummy Alter } 65+i\end{array}$ & $\begin{array}{l}-0,814 \\
(0,877)\end{array}$ & $\begin{array}{l}-0,789^{* * *} \\
(0,256)\end{array}$ \\
\hline $\begin{array}{l}\text { Dummy stationär }{ }_{j} \text { * } \\
\text { Dummy weiblich }{ }_{i}\end{array}$ & $\begin{array}{l}0,010 \\
(0,830)\end{array}$ & $\begin{array}{l}-0,161 \\
(0,240)\end{array}$ \\
\hline In Ausgaben ${ }_{i}$ & $\begin{array}{l}0,205^{* * *} \\
(0,035)\end{array}$ & $\begin{array}{l}0,955^{\star * *} \\
(0,005)\end{array}$ \\
\hline Konstante & $\begin{array}{l}-4,843^{*} \\
(2,610)\end{array}$ & $\begin{array}{l}-3,179^{* * *} \\
(0,570)\end{array}$ \\
\hline Beobachtungen & \multicolumn{2}{|l|}{300.509} \\
\hline
\end{tabular}

Tabelle 7 (Fortsetzung)

\begin{tabular}{lcl}
\hline Unabhängige Variablen & $\begin{array}{l}\text { Koeffizienten } \\
\text { Logit-Modell } \\
\text { (Standardfehler) }\end{array}$ & $\begin{array}{l}\text { Koeffizienten } \\
\text { Zähldaten- } \\
\text { modell } \\
\text { (Standard- } \\
\text { fehler) }\end{array}$ \\
\hline Log Likelihood & $-26.902,59$ \\
AIC & $53.901,19$ \\
\hline Anmerkung**** & &
\end{tabular}

keinen signifikanten Einfluss mehr. Es kann zwar belegt werden, dass Liefergebühren im Lebensmittel-Onlinehandel attraktivitätsmindernd wirken, jedoch nicht, dass - wenn der jeweilige Anbieter gewählt wird - geringere Ausgaben getätigt werden. Dies ist insofern nachvollziehbar, als dass diese Lieferkosten als Pauschale unabhängig von der Bestellmenge berechnet werden (abgesehen davon, dass es in der Regel einen Mindestbestellwert gibt) und dementsprechend sogar eher einen Anreiz bieten, bei einmalig bezahlten Versandpauschalen den Einkauf zu vergrößern.

Die Kanalwahl wurde in diesem Multi-Channel-Modell über Interaktionsterme des psychographischen Konstruktes der Online-Affinität modelliert. Hierbei wird das Ergebnis aus der Experimentalstudie von Schmid und Axhausen (2019) bestätigt: Der Koeffizient der Interaktion der Dummyvariable für stationäre Betriebe und der latenten Variablen (LV) ist signifikant negativ. Je online-affiner ein Konsument also ist, desto geringer ist die Wahrscheinlichkeit, den Einkauf bei einem stationären Anbieter zu vollziehen. Die Kanalwahl wird demnach (auch) durch Einstellungsmuster erklärt. Diese sind nicht (notwendigerweise) altersund geschlechtsabhängig, was sich daran messen lässt, dass auch zwei Kontrollvariablen für Altersgruppen bzw. das Geschlecht sowie deren Interaktion mit der o. g. Dummyvariablen ins Modell integriert wurden. Hierbei zeigen sich allerdings überwiegend keine signifikanten Effekte. Abgesehen von diesen Kanaleffekten zeigt sich, dass sich durch die Integration der Online-Kaufentscheidung in das Modell die Zusammenhänge zwischen den erklärenden Variablen (z. B. Sortiment, Erreichbarkeit) und dem Einkaufsverhalten gegenüber der ersten Modellvariante nicht grundlegend ändern.

\subsection{Stärken und Schwächen der Untersuchung}

Diese Studie stellt den ersten Ansatz dar, stationäre und Online-Anbieter sowie die Cross-Channel-Integration von Verkaufsstellen in ein quantitatives Modell der Einkaufsstättenwahl zu integrieren. Diese Modellfamilie entstammt dem Kontext der Standorttheorie des Einzelhandels, in der nur stationäre Anbieter berücksichtigt werden. Die Ergebnisse 
zeigen die Relevanz von Aspekten des Sortiments sowie der Transaktionskosten beim Einkauf auf, wobei deutlich wird, dass die grundsätzliche Logik der traditionellen Standorttheorie bzw. der Modelle der Einkaufsstättenwahl auf das Einkaufsverhalten im Multi-/Cross-Channel-Kontext übertragbar ist. Derartige Modelle sind von großer Relevanz sowohl in der betrieblichen Standortanalyse als auch in der Verträglichkeitsbeurteilung von Einzelhandelsvorhaben im Rahmen der Bauleitplanung bzw. Raumordnung. Dass mehrere Kaufkanäle hier integrierbar sind, kann die Aussagekraft jener Modelle aufwerten.

Weiterhin stellt diese Arbeit eine direkte Überprüfung von Zusammenhängen dar, die bisher nur in experimentellen Studien (Stated-choice-Befragungsexperimente) aufgearbeitet, hier aber anhand von realen Einkaufsentscheidungen untersucht wurden. In dieser Hinsicht konnte gezeigt werden, dass die Präferenz des Kaufkanals (auch) durch individuelle Einstellungen erklärt wird. ,Online-Affinität ${ }^{*}$ ist somit keine reine Frage des Alters, sondern sowohl demographische als auch psychographische Merkmale leisten voneinander unabhängig einen Erklärungsbeitrag zum tatsächlichen Einkaufsverhalten.

Eine weitere Stärke der Untersuchung liegt in ihrer Repräsentativität. In den meisten Studien zum Kanalwahlverhalten wird eine eingeschränkte Stichprobe verwendet (z. B. nur Internetnutzer/-innen, Multi-Channel-Kunden, Kunden eines spezifischen Unternehmens). Demgegenüber basieren die hiesigen Ergebnisse auf einer Zufallsstichprobe der Gesamtbevölkerung.

Zwar ist die Spaltung des Konsumentenverhaltens in eine grundsätzliche Entscheidung (Einkaufsstättenwahl) und die Intensität der Einkaufsbeziehungen (Ausgaben) eine Stärke des Hurdle-Modellansatzes, jedoch ergeben sich daraus wiederum Schwierigkeiten in der Interpretation. Erstens trifft das Modell keinerlei Aussage darüber, ob diese Entscheidungen tatsächlich - das heißt in der konsumentenseitigen Abwägung der Alternativen - hierarchisch bzw. sukzessiv sind. Zweitens liefern bestehende Theorien und Modelle der Einkaufsstättenwahl keine explizite Erklärung für Unterschiede zwischen den Wirkungen auf die Einkaufsstättenwahl und die Kunden-/Kaufkraftzuflüsse. Beispielsweise setzt das (aggregierte) Huff-Modell implizit Einkaufsentscheidungen und Marktanteile gleich.

Weiterhin muss berücksichtigt werden, dass sich diese Untersuchung auf bestimmte Deutungen und Operationalisierungen etablierter Konzepte bezieht, die vorherigen, vor allem experimentellen Arbeiten entlehnt sind. Damit geht einher, dass ein Oberbegriff wie ,Transaktionskosten " oder ein Konstrukt wie eine psychographisch definierte ,OnlineAffinität ${ }^{*}$ auch nur in diesem Sinne angewendet wird, wobei klargestellt werden muss, dass hier keine vollumfängliche Berücksichtigung aller relevanten Aspekte erfolgte und er- folgen konnte. Beispielsweise ist ,Online-Affinität ' bewusst und vorsätzlich aus einer bestehenden Studie abgeleitet worden, wobei Risikoaversion und ethische Fragestellungen in den Vordergrund gerückt wurden, dabei aber andere Aspekte (z. B. Convenience-Orientierung) außer Acht gelassen wurden. Hier sind nachfolgende Studien gefragt, um psychographische Merkmale von Konsumenten breiter zu fassen und deren Einfluss auf das räumliche Einkaufsverhalten zu prüfen.

Die Aussagekraft im Hinblick auf reales Einkaufsverhalten im Lebensmitteleinzelhandel ist außerdem in zwei Punkten eingeschränkt: Abgesehen davon, dass die räumliche Nähe zu konkurrierenden Anbietern anderer Betriebsformen berücksichtigt wurde, wurden keine weiteren möglichen Wegekopplungen in das Modell einbezogen. Insbesondere beim Lebensmittelkauf sind aber Kopplungen beispielsweise mit dem Arbeitsweg als durchaus relevant anzunehmen, was den tatsächlichen, hier berücksichtigten, Effekt der Fahrtzeit zwischen Wohnort und Einkaufsstätte abschwächt. Im Hinblick auf die Berücksichtigung des CrossChannel-Lebensmitteleinkaufs ist ferner darauf hinzuweisen, dass in der Untersuchung nur die Click-and-collectOption als (angenommenes) Attraktivitätsmerkmal von Lebensmittelmärkten berücksichtigt wurde. $\mathrm{Ob}$ und, wenn ja, bei welchen Einkäufen tatsächlich mittels click and collect eingekauft wurde, ist hingegen nicht erfasst worden.

\section{Schlussfolgerungen}

Es lässt sich anhand der Ergebnisse eine vorsichtige Schlussfolgerung zur tatsächlichen Bedeutung des LebensmittelOnlinehandels als zusätzlichem Kaufkanal ableiten. Werden nur diejenigen Online-Anbieter betrachtet, die im Hinblick auf ihre Sortimentsbreite und -tiefe mit stationären Lebensmittelmärkten vergleichbar sind, zeigt sich - bisher - eine nur sehr geringe Marktbedeutung des digitalen Vertriebsweges. Hierbei muss aber die reziproke Beziehung von Angebot und Nachfrage mitgedacht werden: Ein vollständiger Online-Lieferdienst für Lebensmitteleinkäufe ist im ländlichen der beiden Untersuchungsgebiete - mutmaßlich aufgrund einer Unterschreitung der Tragfähigkeitsschwelle nicht einmal verfügbar. Die Rentabilitätsproblematik stellt, nicht zuletzt durch die Voraussetzungen für den Transport, ein besonderes Hemmnis für den Lebensmittel-Onlinehandel im ländlichen Raum dar (Dannenberg/Dederichs 2019: 18-19). Zudem muss aber auch die soziale Funktion des Lebensmitteleinkaufs bedacht werden. Persönliche Kontakte während des Einkaufens können ein Grund für die Wahl des Kaufkanals sein und werden daher immer wieder als wichtiger Grund für die Aufrechterhaltung stationärer Nahver- 
sorgungsangebote angeführt (Kokorsch/Küpper 2019: 14$15)$.

Dass der Onlinehandel als konkurrierender Kaufkanal (noch) keine nennenswerte Geltung hat, bedeutet jedoch nicht zwangsweise, dass der stationäre Lebensmitteleinzelhandel nicht von einer Cross-Channel-Integration profitieren kann. Daher wurde auch die Möglichkeit von Clickand-collect-Käufen erstmals in dieser Studie als Determinante des (räumlichen) Einkaufsverhaltens geprüft. Das Ergebnis ist allerdings ernüchternd, denn augenscheinlich profitieren Verbrauchermärkte hiervon in der Regel nicht. Was zunächst überrascht, lässt sich allerdings zumindest zu einem Teil mit den Argumenten von Dannenberg, Franz und Lepper (2016: 150-151) erklären: Einerseits verhindert eine Vorbestellung die persönliche Begutachtung der gewünschten Lebensmittel. Es ist anzunehmen, dass die meisten Kundinnen und Kunden einen direkten Kontakt bzw. eine Prüfung der Lebensmittel (sehen, riechen, fühlen usw.) insbesondere bei Frischwaren (z. B. Obst) bevorzugen. Zweitens erhöht click and collect die Flexibilität der Kundinnen und Kunden nur bedingt, denn der Onlineshop ist zwar zeitlich unbegrenzt für Bestellungen verfügbar, die Abholung der Waren ist aber nur zu den regulären Öffnungszeiten der Märkte möglich. Es ist allerdings zu bedenken, dass dieses Angebot zum Zeitpunkt der Erhebung noch relativ neu war; eine steigende Akzeptanz über die Zeit ist nicht auszuschlieBen. Es gibt aber noch eine andere mögliche Erklärung dafür, dass die Einkaufsausgaben in Märkten mit Click-andcollect-Option bzw. bei vorbestellten Einkäufen sogar deutlich niedriger sein können: Die vorherige Bestellung des eigenen Warenkorbs am PC (oder Smartphone) ermöglicht eine zielgerichtete Zusammenstellung der Einkäufe entsprechend vorher festgestellter Bedarfe. Durch Reize am point of sale ausgelöste Spontankäufe, die auch im Lebensmitteleinzelhandel einen relevanten Anteil der Einkäufe ausmachen (Silberer 2013: 80-82), finden bei dieser Form des Lebensmitteleinkaufs nicht statt.

Zusammengefasst zeigen die hiesigen Ergebnisse, dass keinesfalls von einem Trend zur ,digitalen Nahversorgung * gesprochen werden kann. Es ist allerdings denkbar, dass sich die Kanalpräferenzen mittel- und langfristig leicht verschieben, wenn eine heutzutage schon online-affinere Konsumentengeneration in ein Alter bzw. einen Lebensabschnitt kommt, in dem ihre Mobilität eingeschränkt ist (Mensing/ Neiberger 2018: 15-16). Weiterhin stellt sich die Frage, wie sich die Corona-Krise ab März 2020 - die hier verwendeten Daten stammen von 2019 - auf das Einkaufsverhalten auswirkt. Im ersten Halbjahr 2020 wurde ein Wachstum von $9,2 \%$ im deutschen Onlinehandel gegenüber dem Vorjahreszeitraum festgestellt. Im zweiten Quartal 2020 ist eine besonders starke Steigerung von 16,5\% gegenüber dem ersten Quartal 2019 verzeichnet worden, was insbesondere den Le-
bensmittel-Onlinehandel mit einem Umsatzwachstum um $89,4 \%$ betrifft. $^{2} \mathrm{Ob}$ es sich hierbei um einen temporären oder nachhaltigen Effekt handelt, kann noch nicht abschließend beurteilt werden. Es ist daher sinnvoll und notwendig, derartige Untersuchungen zu wiederholen.

Die Relevanz des digitalen Vertriebs wird im Lebensmitteleinzelhandel, wenn auch auf relativ geringem Niveau, definitiv steigen. Daher empfiehlt es sich, den LebensmittelOnlinehandel in Planungsprozessen zumindest ,mitzudenken'. Perspektivisch könnte dies beispielsweise dadurch gewährleistet werden, dass der Lebensmittel-Onlinehandel auch in Verträglichkeitsbeurteilungen berücksichtigt wird. Diese basieren schließlich auf Abschätzungen mithilfe von quantitativen Modellen der Einkaufsstättenwahl (MüllerHagedorn 2020: 116-123) und die vorliegende Untersuchung zeigt, dass Online-Anbieter in eben jene Modelle integriert werden können.

Abseits der Berücksichtigung des Onlinehandels bestätigen die Ergebnisse die theoretischen Aussagen und empirischen Erkenntnisse zum Einkaufsverhalten im stationären Lebensmitteleinzelhandel aus früheren Studien. Dies betrifft insbesondere die herausragende Bedeutung der Erreichbarkeit von Lebensmittelmärkten bei der Einkaufsstättenwahl. Dies bedeutet keinesfalls, dass Konsumentinnen und Konsumenten im Sinne des Zentrale-OrteBasismodells stets den jeweils nächsten Angebotsstandort aufsuchen, sehr wohl aber, dass die Entfernung bzw. Wegezeit in der implizit stattfindenden Abwägung zwischen den Pro- und Contra-Argumenten der Einkaufsalternativen das höchste Gewicht hat. Die Fokussierung von Erreichbarkeitsaspekten sowohl bei der wissenschaftlichen Analyse als auch bei der normativen Seite der Raumplanung ist daher sinnvoll und notwendig, wenn eine ,ausreichende Nahversorgung operationalisiert werden soll.

Förderhinweis Gefördert durch die Deutsche Forschungsgemeinschaft (DFG) - Projektnummer 402130768.

Danksagung Der DFG sei für die finanzielle Förderung des Projekts gedankt. Der Autor dankt weiterhin den beiden studentischen Hilfskräften Anne Auinger und Anjulie Kappler für ihre unersetzliche Mitarbeit in diesem Projekt. Dank gebührt zudem den Ansprechpartner/-innen mehrerer Handelsunternehmen sowie dem Regionalverband Mittlerer Oberrhein und mehrerer Gemeinden für die Bereitstellung von Standortdaten. 


\section{Literatur}

Anders, S. (2018): Standortkonkurrenz von Lebensmittelmärkten. Frühzeitige Abschätzung und Bewertung der Auswirkungen neuer Märkte. In: Raumforschung und Raumordnung | Spatial Research and Planning 76, 4, 297-308. https://doi.org/10.1007/s13147-018-0538-z

Beckers, J.; Cárdenas, I.; Verhetsel, A. (2018): Identifying the geography of online shopping adoption in Belgium. In: Journal of Retailing and Consumer Services 45, 3341. https://doi.org/10.1016/j.jretconser.2018.08.006

Bezes, C. (2016): Comparing online and in-store risks in multichannel shopping. In: International Journal of Retail and Distribution Management 44, 3, 284-300. https:// doi.org/10.1108/IJRDM-02-2015-0019

Boniversum (2018): „Click \& Collect“ - Verbreitung und Nutzung. Boniversum Verbraucherumfrage 11/2018. Neuss. https://www.boniversum.de/wp-content/uploads/ 2018/11/Boniversum_bevh_Studie_Click-Collect.pdf (05.01.2021).

Briesch, R. A.; Chintagunta, P. K.; Fox, E. J. (2009): How Does Assortment Affect Grocery Store Choice? In: Journal of Marketing Research 46, 2, 176-189. https://doi. org/10.1509/jmkr.46.2.176

Burkolter, D.; Kluge, A. (2011): Online consumer behavior and its relationship with socio-demographics, shopping orientations, need for emotion, and fashion leadership. In: Journal of Business and Media Psychology 2, 2, 2028.

Cameron, A. C.; Trivedi, P. K. (2005): Microeconometrics: Methods and applications. Cambridge. https://doi.org/ 10.1017/CBO9780511811241

Cao, X.; Chen, Q.; Choo, S. (2013): Geographic Distribution of E-Shopping: Application of Structural Equation Models in the Twin Cities of Minnesota. In: Transportation Research Record 2383, 1, 18-26. https://doi.org/10. 3141/2383-03

Chintagunta, P. K.; Chu, J.; Cebollada, J. (2012): Quantifying Transaction Costs in Online/Off-line Grocery Channel Choice. In: Marketing Science 31, 1, 96-114. https:// doi.org/10.1287/mksc.1110.0678

Clarke, G.; Thompson, C.; Birkin, M. (2015): The emerging geography of e-commerce in British retailing. In: Regional Studies, Regional Science 2, 1, 371-391. https://doi. org/10.1080/21681376.2015.1054420

Crowley, F.; Eakins, J.; Jordan, D. (2012): Participation, expenditure and regressivity in the Irish lottery: Evidence from Irish household Budget Survey 2004/2005. In: The Economic and Social Review 43, 2, 199-225.

Dannenberg, P.; Dederichs, S. (2019): Online-Lebensmittelhandel in ländlichen Räumen. Hemmnisse einer Expansion des Onlinehandels mit Lebensmitteln aus der Per- spektive unterschiedlicher Akteure in Deutschland. In: RaumPlanung 202, 3/4, 16-21.

Dannenberg, P.; Franz, M.; Lepper, A. (2016): Online einkaufen gehen - Einordnung aktueller Dynamiken im Lebensmittelhandel aus Perspektive der geographischen Handelsforschung. In: Franz, M.; Gersch, I. (Hrsg.): Online-Handel ist Wandel. Mannheim, 133-156. = Geographische Handelsforschung 24.

Ganesh, J.; Reynolds, K. E.; Luckett, M.; Pomirleanu, N. (2010): Online Shopper Motivations, and e-Store Attributes: An Examination of Online Patronage Behavior and Shopper Typologies. In: Journal of Retailing 86, 1, 106115. https://doi.org/10.1016/j.jretai.2010.01.003

Greene, W. H. (2012): Econometric Analysis. Harlow.

Hahn Gruppe (2019): Retail Real Estate Report Germany 2019/2020. https://www.hahnag.de/index.php?eID=tx nawsecured $\& \mathrm{u}=0 \& \mathrm{~g}=0 \& \mathrm{t}=1610535303 \&$ hash $=68 \mathrm{~b} 2 \mathrm{~b}$ 889244280ad0574de9b6fd48475f3b0652f\&

file $=$ media/hahn/Downloads_PDF/Publikationen/ Research/Hahn_Retail_Real_Estate_Report_2019_ web.pdf (05.01.2021).

Haubach, C.; Held, B. (2015): Ist ökologischer Konsum teurer? Ein warenkorbbasierter Vergleich. In: Wirtschaft und Statistik 1, 41-55.

HDE - Handelsverband Deutschland; IFH - IFH Köln GmbH (2018): Handelsreport Lebensmittel. Fakten zum Lebensmitteleinzelhandel. Berlin.

HDE - Handelsverband Deutschland; IFH - IFH Köln GmbH (2020): Online-Monitor 2020. Berlin.

Hillier, A.; Smith, T. E.; Whiteman, E. D.; Chrisinger, B. W. (2017): Discrete Choice Model of Food Store Trips Using National Household Food Acquisition and Purchase Survey (FoodAPS). In: International Journal of Environmental Research and Public Health 14, 10, 1133. https://doi.org/10.3390/ijerph14101133

Hsiao, M.-H. (2009): Shopping mode choice: Physical store shopping versus e-shopping. In: Transportation Research Part E: Logistics and Transportation Review 45, 1, 8695. https://doi.org/10.1016/j.tre.2008.06.002

Huff, D. L. (1962): Determination of Intra-Urban Retail Trade Areas. Los Angeles.

Kläsgen, M. (2019): Wie klimaschädlich ist der Onlinehandel? In: Süddeutsche Zeitung vom 4. Mai 2019. https:// www.sueddeutsche.de/wirtschaft/online-shopping-co2klima-laden-1.4429396 (05.01.2021).

Kokorsch, M.; Küpper, P. (2019): Trends der Nahversorgung in ländlichen Räumen. Braunschweig. = Thünen Working Paper 126.

Küpper, P.; Scheibe, C. (2015): Steuern oder fördern? Die Sicherung der Nahversorgung in den ländlichen Räumen Deutschlands und Südtirols im Vergleich. In: Raumfor- 
schung und Raumordnung 73, 1, 45-58. https://doi.org/ 10.1007/s13147-014-0326-3

Lademann, R. P. (2007): Zum Einfluss von Verkaufsfläche und Standort auf die Einkaufswahrscheinlichkeit. In: Schuckel, M.; Toporowski, W. (Hrsg.): Theoretische Fundierung und praktische Relevanz der Handelsforschung. Wiesbaden, 143-162. https://doi.org/10.1007/ 978-3-8350-9535-9_8

Lademann, R.P. (2013): Wettbewerbsökonomische Grundlagen des Betriebsformenwettbewerbs im Lebensmitteleinzelhandel. In: Riekhof, H.-C. (Hrsg.): Retail Business. Perspektiven, Strategien, Erfolgsmuster. Wiesbaden, 3-30. https://doi.org/10.1007/978-3-8349-4555-6_ 1

Mensing, M. (2018): Lebensmittel-Onlinehandel - Alternative zur zukünftigen Versorgung der Bevölkerung ländlicher Räume? Dissertation, Rheinisch-Westfälische Technische Hochschule Aachen. https://doi.org/10. 18154/RWTH-2019-02683

Mensing, M.; Neiberger, C. (2016): Mapping E-Commerce - regionale Unterschiede im Online-Einkaufsverhalten deutscher Verbraucher. In: Franz, M.; Gersch, I. (Hrsg.): Online-Handel ist Wandel. Mannheim, 109-131. = Geographische Handelsforschung 24.

Mensing, M.; Neiberger, C. (2018): Onlinehandel mit Lebensmitteln - Eine Möglichkeit zur Lösung der Versorgungsprobleme im ländlichen Raum? In: Europa Regional 26, 1, 2-19.

Müller-Hagedorn, L. (2020): Einzelhandelsgutachten sind eine schwierige Dienstleistung. In: Roth, S.; Horbel, C.; Popp, B. (Hrsg.): Perspektiven des Dienstleistungsmanagements. Wiesbaden, 105-125. https://doi.org/10.1007/ 978-3-658-28672-9_7

Nielsen - The Nielsen Company (Germany) (2016): Deutschland 2016. Handel, Verbraucher, Werbung. https://www.nielsen.com/wp-content/uploads/sites/3/ 2019/04/Nielsen-Booklet_Handel-VerbraucherWerbung_2016.pdf (26.06.2017).

Popkowski Leszczyc, P. T. L.; Sinha, A.; Sahgal, A. (2004): The effect of multi-purpose shopping on pricing and location strategy for grocery stores. In: Journal of Retailing 80, 2, 85-99. https://doi.org/10.1016/j.jretai.2004.04.006

Ren, F; Kwan, M.-P. (2009): The Impact of Geographic Context on E-Shopping Behavior. In: Environment and Planning B: Planning and Design 36, 2, 262-278. https:// doi.org/10.1068/b34014t

Reutterer, T.; Teller, C. (2009): Store format choice and shopping trip types. In: International Journal of Retail and Distribution Management 37, 8, 695-710. https:// doi.org/10.1108/09590550910966196

Rittinger, S. (2013): Cross-Channel Retail Branding. Eine verhaltenswissenschaftliche Untersuchung in Deutsch- land, Frankreich und Großbritannien. Wiesbaden. https:// doi.org/10.1007/978-3-658-03610-2

Schaer, C. (2018): The dark side of Germany's online shopping boom. In: Handelsblatt vom 18. November 2018. https://www.handelsblatt.com/english/ companies/courier-rights-the-dark-side-of-germanysonline-shopping-boom/23694948.html (05.01.2021).

Schmid, B.; Axhausen, K. W. (2019): In-store or online shopping of search and experience goods: A Hybrid Choice approach. In: Journal of Choice Modelling 31, 156-180. https://doi.org/10.1016/j.jocm.2018.03.001

Schröder, H.; Witek, M. (2010): Zur Bedeutung des Katalogkanals (Mailorder-Channel) für einen MultichannelRetailer - Antworten geben die Kaufmotive. In: Ahlert, D.; Kenning, P.; Olbrich, R.; Schröder, H. (Hrsg.): Multichannel-Management. Frankfurt am Main, 73101. = Jahrbuch Vertriebs- und Handelsmanagement 2010/2011.

Silberer, G. (2013): Kaufentscheidungen und Kaufhandlungen am Point-of-Sale - Ein Überblick sowie Stand der Forschung und neuere Befunde. In: Crockford, G.; Ritschel, F.; Schmieder, U. (Hrsg.): Handel in Theorie und Praxis. Wiesbaden, 69-95. https://doi.org/10.1007/9783-658-01986-0_3

Sinus (2018): Die Mehrheit der Deutschen zweifelt am Datenschutz. Pressemitteilung vom 24. Januar 2018. https://www.sinus-institut.de/fileadmin/user_data/sinusinstitut/Bilder/news/Datenschutztag/Pressetext_

Datenschutztag_SINUSYouGov.pdf (08.01.2021).

Statista (2019): Anzahl der Einzelartikel in Lebensmittel-Discountern in Deutschland nach Unternehmen im Jahr 2019. https://de.statista.com/statistik/daten/studie/ 479391/umfrage/artikel-in-lebensmittel-discountern-indeutschland/ (05.01.2021).

Steiger, M. (2017): Multiagentensysteme zur Simulation von Konsumentenverhalten - Untersuchung individuenbasierter Simulationsszenarien zur strategischen Standortplanung im Einzelhandel. Mannheim. = Geographische Handelsforschung 26.

Suárez-Vega, R.; Gutiérrez-Acuña, J. L.; Rodríguez-Díaz, M. (2015): Locating a supermarket using a locally calibrated Huff model. In: International Journal of Geographical Information Science 29, 2, 217-233. https://doi. org/10.1080/13658816.2014.958154

Suel, E.; Polak, J. W. (2018): Incorporating online shopping into travel demand modelling: challenges, progress, and opportunities. In: Transport Reviews 38, 5, 576-601. https://doi.org/10.1080/01441647.2017.1381864

Tihi, B.; Oruc, N. (2012): Competitive Location Assessment - the MCI Approach. In: South East European Journal of Economics and Business 7, 2, 35-49. https:// doi.org/10.2478/v10033-012-0013-7 
Toporowski, W.; Lademann, R. P. (2014): The importance of assortment, pricing, and retail site location for competition in food retailing - Results from marketing research. In: Marketing ZFP - Journal of Research and Management 36, 2, 131-140. https://doi.org/10.15358/ 0344-1369_2014_2_131

Wiegandt, C.-C.; Baumgart, S.; Hangebruch, N.; Holtermann, L.; Krajewski, C.; Mensing, M.; Neiberger, C.; Osterhage, F.; Texier-Ast, V.; Zehner, K.; Zucknik, B. (2018): Determinanten des Online-Einkaufs - eine empirische Studie in sechs nordrhein-westfälischen Stadtregionen. In: Raumforschung und Raumordnung | Spatial Research and Planning 76, 3, 247-265. https://doi.org/ 10.1007/s13147-018-0532-5

Wieland, T. (2015): Räumliches Einkaufsverhalten und Standortpolitik im Einzelhandel unter Berücksichtigung von Agglomerationseffekten: Theoretische Erklärungsansätze, modellanalytische Zugänge und eine empirisch-ökonometrische Marktgebietsanalyse anhand eines Fallbeispiels aus dem ländlichen Raum Ostwestfalens/ Südniedersachsens. Mannheim. = Geographische Handelsforschung 23.

Wieland, T. (2018): A Hurdle Model Approach of Store
Choice and Market Area Analysis in Grocery Retailing. In: Papers in Applied Geography 4, 4, 370-389. https:// doi.org/10.1080/23754931.2018.1519458

Wooldridge, J. M. (2002): Econometric Analysis of Cross Section and Panel Data. Cambridge.

Zaharia, S.; Hackstetter, T. (2017): Segmentierung von Onlinekäufern auf Basis ihrer Einkaufsmotive. In: Deutscher Dialogmarketing Verband (Hrsg.): Dialogmarketing Perspektiven 2016/2017. Tagungsband 11. wissenschaftlicher interdisziplinärer Kongress für Dialogmarketing, 45-72. Wiesbaden. https://doi.org/10.1007/9783-658-16835-3_3

Zhai, Q.; Cao, X.; Mokhtarian, P. L.; Zhen, F. (2017): The interactions between e-shopping and store shopping in the shopping process for search goods and experience goods. In: Transportation 44, 885-904. https://doi.org/ 10.1007/s11116-016-9683-9

Zhen, F.; Du, X.; Cao, J.; Mokhtarian, P. L. (2018): The association between spatial attributes and e-shopping in the shopping process for search goods and experience goods: Evidence from Nanjing. In: Journal of Transport Geography 66, 291-299. https://doi.org/10.1016/j. jtrangeo.2017.11.007 